\title{
AN APPROACH TO HOMOTOPY CLASSIFICATION OF LINKS
}

\author{
J. P. LEVINE
}

\begin{abstract}
A reformulation and refinement of the $\bar{\mu}$-invariants of Milnor are used to give a homotopy classification of 4 component links and suggest a possible general homotopy classification. The main idea is to use the (reduced) group of a link and its "geometric" automorphisms to define the precise indeterminacy of these invariants.
\end{abstract}

Introduction. In 1954, Milnor [M] introduced the concept of link homotopy, a notion considerably weaker than the usual notion of link type. In fact it is also weaker than the relations of PL and topological isotopy and $I$-equivalence introduced later (see $[\mathbf{R}, \mathbf{G o}, \mathbf{G} \mathbf{1}]$ ), but equivalent to the apparently weaker relation which asks for disjoint singular cylinders in $I \times S^{3}$ connecting the links (see [Li]). Recently link homotopy has come up in a reformulation of the 4-dimensional topological surgery conjecture (see [FL]).

Unlike usual link type the classification problem for link homotopy seems quite tractable-in [M] Milnor gives a complete homotopy classification of links with 3 or fewer components, and shows that a link is homotopically trivial if and only if a certain collection of numerical invariants is defined and vanishes-alternatively this can be interpreted in terms of the reduced link group $\mathcal{G}(L)$ defined in $[\mathbf{M}]$ (see below).

The numerical homotopy invariants used in $[\mathbf{M}]$ are part of a wider collectionthe $\bar{\mu}$-invariants - which appear in [M1] and are there shown to be invariants of topological isotopy. In $[\mathbf{S}]$ they are shown to be invariants of topological $I$ equivalence, but are not, except for the subset used in $[\mathbf{M}]$, homotopy invariants. Although the $\bar{\mu}$-invariants do not serve to classify links under $I$-equivalence, it is still an open question whether a link with trivial $\bar{\mu}$-invariants is cobordant to a boundary link. A boundary link is one whose components bound disjoint surfaces in $S^{3}$-they have trivial $\bar{\mu}$-invariants and include all split links (links whose components lie in disjoint balls in $S^{3}$ ) and parallel links (links formed by several "parallel" copies of a single knot). As already mentioned, the homotopy $\bar{\mu}$-invariants are trivial if and only if the link is homotopy trivial but it is not true that any links with the same $\bar{\mu}$-invariants are homotopic $[\mathbf{L}]$ - their indeterminacy is too large.

In $[\mathbf{L}]$ a classification of links up to surgery equivalences is given using certain of the homotopy $\bar{\mu}$-invariants with a refinement of their indeterminacy. In fact, it seems implicit in $[\mathbf{M}]$ that one should be able to use the $\bar{\mu}$-invariants to give a general homotopy classification. It is shown in $[\mathbf{M}, \mathbf{P}]$ that the $\bar{\mu}$-invariants

Received by the editors December 12, 1986.

1980 Mathematics Subject Classification (1985 Revision). Primary 57M25.

Partially supported by the National Science Foundation. 
with their original indeterminacy already classify the "almost 2-trivial" linksthose which become homotopy trivial when any two components are removed.

It is the aim of this paper to initiate a program for solving this problem. We will obtain a formulation of a new and much more delicate indeterminacy for the $\bar{\mu}$ invariants which allows us to distinguish many links which are not distinguished by the old indeterminacy. Furthermore it seems hopeful that this may be the correct indeterminacy for complete homotopy classification. We will show this to be true for 4-component links (our main application) and a general class of links containing the "almost 2-trivial" links.

The primary tool in this study is the group $\mathcal{G}(L)$, a certain quotient of the fundamental group $G(L)$ of the complement of the link $L$ defined in $[\mathbf{M}] \cdot \mathcal{G}(L)$, together with a "peripheral structure" consisting of elements of defined by meridians and longitudes of the link components, is shown in $[\mathbf{M}]$ to be a homotopy invariant up to a certain explicit indeterminacy in the peripheral structure. We will show that this indeterminacy in the peripheral structure can be substantially reduced. Corresponding to the results mentioned above we will see that $\mathcal{G}(L)$, with this more delicate peripheral structure, provides a homotopy classification within the same class of links. In fact these two classification schemes are generally equivalent.

The approach we take (begun in $[\mathbf{M}]$ ) is to study a link $L$ by taking one of its components to represent a conjugacy class of elements $\alpha$ in $\mathcal{G}(K)$, where $K$ is the sublink of $L$ defined by the remaining components. The crucial observation is that the homotopy class of $L$ is determined by $K$ together with the orbit of $\alpha$ in $\mathcal{G}(K)$ under automorphisms induced by homotopies of $K$ to itself (we call these geometric automorphisms). Thus the classification problem reduces, in a recursive manner, to the problem of deciding which automorphisms are geometric. The automorphisms preserving the peripheral structure of $\mathcal{G}(K)$, which we call strong, certainly contain the geometric ones. The orbit of $\alpha$ under strong automorphisms can be used to define the new indeterminacy of the $\bar{\mu}$-invariants; it is the correct indeterminacy for classification if all strong automorphisms are geometric. This is proved to be the case for 3-component links and "almost trivial" links. In fact the larger and simpler group of automorphisms of $\mathcal{G}(K)$ which preserve the meridians (called special), can be used to define a simpler indeterminacy for the $\bar{\mu}$-invariants which, although too large to have hope of classification, is often smaller than the old indeterminacy.

This paper consists of three parts. In the first part we define the peripheral structure in $\mathcal{G}(L)$, demonstrate its homotopy invariance, and rephrase the classification problem as a question of determining the geometric automorphisms. The $\bar{\mu}$-invariants arise as exponents in a representation of an element of $\mathcal{G}(K)$ as a monomial in basic commutators of the meridians. The indeterminacy comes from the change in such a representation induced by relations in $\mathcal{G}(K)$ and geometric automorphisms. Our approach to the $\bar{\mu}$-invariants is technically different from Milnor's in the use of basic commutators rather than the Magnus expansion used in $[\mathbf{M}, \mathbf{M 1}]$.

In the second part we show that, for an almost trivial link, every strong automorphism is geometric. This follows from one general technique for constructing geometric automorphisms and the observation that for these links they generate all the special automorphisms. 
In the third part, we show that, for 3-component links, all strong automorphisms are geometric. Here we find that special automorphisms are not necessarily strong and, if fact, that there are automorphisms which preserve the old peripheral structure considered in $[\mathbf{M}]$ but are not strong.

In this context we present several examples of nonhomotopic links with identical $\bar{\mu}$-invariants (in their original indeterminacy). In fact, we are able to find links whose reduced groups are isomorphic, preserving meridians, but are not homotopic.

The argument here is rather technical but one interesting feature is the nontrivial use of a rather obvious and seemingly innocuous symmetry which relates the geometric automorphisms of the various sublinks of a given link. Although the explicit general description of the indeterminacy in the $\bar{\mu}$-invariants is unwieldy, in some special cases we are able to obtain a neat statement which exhibits some new invariants that are independent of the $\bar{\mu}$-invariants in their original indeterminacy.

The numbering of the theorems, etc. will be according to the section in which they appear.

\section{PART I}

1. We will define a link of multiplicity $m$ to be an ordered collection of $m$ oriented, smoothly imbedded disjoint circles in $S^{3}$. A sublink is any subcollection with the induced order. The principal sublink is the sublink of multiplicity $m-1$ obtained by deleting the last component. An (ambient) isotopy between links $L_{0}$ and $L_{1}$ is an orientation-preserving diffeomorphism of $S^{3}$ which maps $L_{0}$ onto $L_{1}$ preserving the order and orientation of the components. We will generally omit the word ambient when referring to an isotopy and we warn the reader, therefore, not to confuse this with the notion of PL or topological isotopy which is not ambient. The group of the link $L$, denoted $G(L)$, is the fundamental group $S^{3}-L$. A peripheral structure on $L$ is a collection $\left(\mu_{1}, \lambda_{1}\right), \ldots,\left(\mu_{m}, \lambda_{m}\right)$, where $m$ is the multiplicity of $L$ and $\mu_{i}, \lambda_{i}$ are meridians and longitudes of $L$ defined explicitly as follows. Choose oriented closed curves $m_{i}, l_{i}$ on the boundary $\dot{T}_{i}$ of a tubular neighborhood $T_{i}$ of the $i$ th component $L(i)$ of $L$, determined up to homotopy by the properties: $m_{i}$ is null-homotopic in $T_{i}$ and has linking number +1 with $L(i) ; l_{i}$ is homotopic to $L(i)$ in $T_{i}$ and has linking number 0 with $L(i)$-recall that $L(i)$ is oriented. We may assume $l_{i}$ and $m_{i}$ intersect transversally at a single point $x_{i} \in \dot{T}_{i}$. For any path $\gamma_{i}$ in $S^{3}-L$ from the basepoint to $x_{i}$, the elements $\mu_{i}$ and $\lambda_{i}$ in $G(L)$, represented by $\gamma_{i} m_{i} \gamma_{i}^{-1}$ and $\gamma_{i} l_{i} \gamma_{i}^{-1}$, will be referred to as a compatible $i$-meridian and $i$-longitude pair; $\gamma_{i}$ will be called a stem for the pair $\left(\mu_{1}, \lambda_{i}\right)$. Choice of a different stem will change $\left(\mu_{i} \lambda_{i}\right)$ to $\left(\mu_{i}^{\prime}, \lambda_{i}^{\prime}\right)$ where $\mu_{i}^{\prime}=g_{i} \mu_{i} g_{i}^{-1} \lambda_{i}^{\prime}=g_{i}^{\prime} \lambda_{i} g_{i}^{-1}$ for some $g_{i} \in G(L)$. Two peripheral structures $\left\{\mu_{i}, \lambda_{i}\right\}$ and $\left\{\mu_{i}^{\prime}, \lambda_{i}^{\prime}\right\}$ are conjugate if, for some $g \in G(L), \mu_{i}^{\prime}=g \mu_{i} g^{-1}$, $\lambda_{i}^{\prime}=g \lambda_{i} g^{-1}$. The structured group of $L$ is $G(L)$ together with all the peripheral structures. If $L$ and $L^{\prime}$ are isotopic links then it is clear that $G(L)$ and $G\left(L^{\prime}\right)$ are isomorphic as structured groups i.e. there is an isomorphism $\phi: G(L) \rightarrow G\left(L^{\prime}\right)$ such that $\left\{\mu_{i}, \lambda_{i}\right\}$ is a peripheral structure on $L$, then $\left\{\phi\left(\mu_{i}\right), \phi\left(\lambda_{i}\right)\right\}$ is a peripheral structure on $L^{\prime}$.

2. Suppose $L_{0}, L_{1}$ are links which are defined by smooth imbeddings $i_{0}, i_{1}: \Sigma \rightarrow$ $S^{3}$, where $\Sigma$ is the disjoint union of $m$ circles $S_{1}+S_{2}+\cdots+S_{m}$. Then $L_{0}$ and $L_{1}$ are homotopic if there is a homotopy $i_{t}: \Sigma \rightarrow S^{3}, 0 \leq t \leq 1$, such that $i_{t}\left(S_{i}\right)$ and $i_{t}\left(S_{j}\right)$ are disjoint for any $i \neq j$ and $0 \leq t \leq 1$. We will use the alternative 

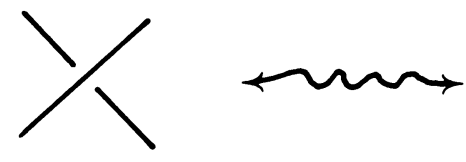

FIGURE 1

characterization of homotopy as the equivalence relation among links generated by link isomorphisms and local crossing changes of arcs from the same link component (see Figure 1 and $[\mathbf{M}]$ ).

We recall the definition of $\mathcal{G}(L)$ from $[\mathbf{M}]$. Define $J(L) \subseteq G(L)$ to be the normal subgroup generated by all elements of the form $\left[\mu_{i}, \mu_{i}^{\prime}\right]$, where $\mu_{i}, \mu_{i}^{\prime}$ are $i$-meridians for any $i=1, \ldots, m$. Then $\mathcal{G}(L)=G(L) / J(L)$ is the reduced group of $L$. We also consider peripheral structures on $\mathcal{G}(L)$. If $\left\{\mu_{i}, \lambda_{i}\right\}$ is the image in $\mathcal{G}(L)$ of a compatible $i$-meridian and $i$-longitude pair and $\bar{\theta}_{i}$ is the coset of $\lambda_{i}$ modulo $\bar{N}_{i}(L)$, the normal subgroup of $\mathcal{G}(L)$ generated by an $i$-meridian, then $\left\{\mu_{i}, \bar{\theta}_{i}\right\}$ will be referred to as a preperipheral structure on $\mathcal{G}(L)$. It is proved in $[\mathbf{M}]$ that homotopic links $L_{0}, L_{1}$ yield an isomorphism $\mathcal{G}\left(L_{0}\right) \approx \mathcal{G}\left(L_{1}\right)$ which preserves the preperipheral structures. We will consider the coset $\theta_{i}$ of $\lambda_{i}$ modulo $N_{i}(L)$, the normal subgroup of $\mathcal{G}(L)$ generated by elements of the form $\left[g,\left[g, \mu_{i}\right]\right]$, for any $g \in \mathcal{G}(L)$. Then $\left\{\mu_{i}, \theta_{i}\right\}$ will be called a peripheral structure on $\mathcal{G}(L) . \quad \mathcal{G}(L)$, together with its peripheral structures, is the reduced structured group of $L$. We will show in $\S 4$ that a homotopy of links defines, in a functorial way, an isomorphism of the reduced structured groups.

3. We first recall a theorem from $[\mathbf{M}]$ on the structure of $\mathcal{G}(L)$ with a slightly different presentation.

PROPOSITION 3. Let $L$ be a link and $\left\{\mu_{i}, \lambda_{i}\right\}$ a peripheral structure in $G(L)$. Then $\mathcal{G}(L)$ is generated by $\mu_{1}, \ldots, \mu_{m}$. Furthermore there exist words $w_{1}, \ldots, w_{m}$ in the $\left\{\mu_{i}\right\}$ which represent $\lambda_{i}$ in $\mathcal{G}(L)$ so that a complete set of relations for $\mathcal{G}(L)$ are those of the followng two types:

(i) $\left[\mu_{i}, w_{i}\right]=1, i=1, \ldots, m$.

(ii) $C=1$, where $C$ is any commutator in $\left\{\mu_{i}\right\}$ with repeats.

To explain the meaning of (ii), we make the following definitions A commutator in $\left\{\mu_{i}\right\}$ can be defined recursively:

(a) The commutators of weight one are $\mu_{1}, \ldots, \mu_{m}$.

(b) The commutators of weight $n$ are words $\left[C_{1}, C_{2}\right]$ where $C_{1}, C_{2}$ are distinct commutators of weight $<n$ and $n=$ wt $C_{1}+$ wt $C_{2}$.

Note that there are only a finite number of commutators of any given weight. If $F$ denotes the free group on $\left\{\mu_{i}\right\}$, then the commutators of weight $\geq n$ generate the subgroup $F_{n}$, which is normal. $F=F_{1} \supseteq F_{2} \supseteq \cdots \supseteq F_{n} \supseteq F_{n+1} \supseteq \cdots$ is the lower central series of $F$ and $\bigcap_{n} F_{n}=\{1\}$ (see [MKS, or LS]).

We will say that $\mu_{i}$ occurs $r$ times in a commutator $C$ as follows.

(a) if $C=\mu_{j}$, then $r=1$ if $i=j$ and 0 otherwise.

(b) if $C=\left[C_{1}, C_{2}\right]$ then $r=r_{1}+r_{2}$, where $\mu_{i}$ occurs $r_{s}$ times in $C_{s}, s=1,2$.

We will say that a commutator $C$ has repeats if some $\mu_{i}$ occurs at least twice in $C$. 
PROOF OF Proposition. In light of $[\mathbf{M}, \S 6]$ we need only prove that $J_{1}=J_{2}$ where $J_{1}$ is the normal subgroup of $F$, generated by commutators with repeats and $J_{2}$ is the normal subgroup generated by all elements of the form $\left[\mu_{i}^{\prime}, \mu_{i}^{\prime \prime}\right]$, where $\mu_{i}^{\prime}, \mu_{i}^{\prime \prime}$ are any conjugates of $\mu_{i}$, and $1 \leq i \leq m$. That $J_{1} \subseteq J_{2}$ is clear since any commutator in which $\mu_{i}$ occurs is in the normal closure $\bar{N}_{i}$ of $\mu_{i}$. We will prove that $J_{2} \subseteq J_{1}$ using the notion of basic commutator, which we will need later.

We recall the definition (see $[\mathbf{S}, \mathbf{M K S}]$ ). A set of basic commutators in $\left\{\mu_{i}\right\}$ is an ordered set of commutators $C_{1}, C_{2}, \ldots$ with the following properties:

(1) $C_{i}=\mu_{i}$ if $i=1, \ldots, m$.

(2) wt $C_{i} \leq \mathrm{wt} C_{j}$ if $i \leq j$.

(3) If $k>m$, then $C_{k}=\left[C_{i}, C_{j}\right]$ for some $i<j<k$.

(4) If $C_{j}=\left[C_{r}, C_{s}\right]$ in (3), then $r \leq i$.

(5) Every $\left[C_{i}, C_{j}\right]$ with $i<j$ satisfying (4) is a basic commutator.

We quote the Basis theorem of P. Hall, concerning the lower central series of $F$.

THEOREM 3 (SEE [MKS]). There exists a set of basic commutators, for any $m$. Given any set of basic commutators $C_{1}, C_{2}, \ldots$, then every element of $F / F_{q}$ has a unique representative as a monomial, $C_{1}^{e_{1}} C_{2}^{e_{2}} \cdots C_{n}^{e_{n}}$, where $C_{1}, \ldots, C_{n}$ are all the basic commutators of weight $<q$.

We now show $J_{2} \subseteq J_{1}$. First notice that $F_{m+1} \subseteq J_{1}$, since it is obvious that any commutator of weight $>m$ must have repeats. Now, if $\alpha \in \bar{N}_{i}$ we can write $\alpha$ as a monomial in basic commutators $\bmod F_{m+1}$. If $C$ is any commutator appearing in this monomial then $\mu_{i}$ occurs in $C$. To see this, notice that any set of basic commutators in $\left\{\mu_{1}, \ldots, \mu_{m}\right\}$ determines a set of basic commutators in $\left\{\mu_{1}, \ldots, \hat{\mu}_{i}, \ldots, \mu_{m}\right\}$ by deleting those in which $\mu_{i}$ occurs. If $\mu_{i}$ did not occur in $C$, then $\alpha$ would have a nontrivial representation as a monomial in basic commutators in $F^{\prime}=F / \bar{N}_{i}$, the free group on $\mu_{1}, \ldots, \hat{\mu}_{i}, \ldots, \mu_{m}$. Since $\alpha \in \bar{N}_{i}$, this violates the Hall Basis theorem applied to $F^{\prime}$. Now if $\alpha, \beta \in \bar{N}_{i}$, we can write each as a monomial in basic commutators in which $\mu_{i}$ occurs, $\bmod F_{m+1}$. If we substitute these monomials onto $[\alpha, \beta]$ and expand, we obtain a product of commutators each of which has repeats - since $F_{m+1} \subseteq J_{1}$, we conclude $[\alpha, \beta] \in J_{1}$.

This complete the proof of Proposition 3. We can now denote $J_{1}=J_{2}$ by $J$. At this time we also record, for future use.

LEMMA 3. Let $\left\{C_{i}\right\}$ be a set of basic commutators in $\left\{\mu_{i}\right\}$ and suppose $C_{1}, \ldots$, $C_{n}$ are those of weight $\leq q$. If an element of $J$ has a representation $C_{1}^{e_{1}}, \ldots, C_{n}^{e_{n}}$ modulo $F_{q+1}$, then whenever $e_{i} \neq 0, C_{i}$ must be a commutator with repeats.

To prove this we use the Magnus expansion (see [LS]). This is an isomorphism (denoted $M$ ) of $F$ into the group of units $U$ of the ring $P$ of noncommuting power series in variables $x_{1}, \ldots, x_{n}$, defined by $M\left(\mu_{i}\right)=1+x_{i}, M\left(\mu_{i}^{-1}\right)=1 / 1+x_{i}$. An element of $U$ can be written uniquely in the form $\pm 1+\rho+\xi$ where $\rho$ is homogeneous of degree $>0$ and each term of $\xi$ has degree larger than $\operatorname{deg} \rho$; we call $\rho$ the principal part. We make the following observation. If $C$ is a commutator in $\left\{\mu_{i}\right\}$ of weight $n$, then the principal part $\rho$ of $M(C)$ has degree $n$ and each $x_{i}$ appears in every term of $\rho$ with total degree equal to the number of occurrences of $\mu_{i}$ in $C$. To see this, suppose $C=\left[C_{1}, C_{2}\right]$ where wt $C_{i}=n_{i}(i=1,2) n=n_{1}+n_{2}$. Then $M\left(C_{i}\right)$ has principal part $\rho_{i}$ where $\operatorname{deg} \rho_{i}=n_{i}$ (see [LS]) and one computes that 
$M(C)=1+\rho_{1} \rho_{2}-\rho_{2} \rho_{1}+$ terms of degree $>n$. Since $C \notin F_{n+1}$, we conclude that $\rho_{1} \rho_{2}-\rho_{2} \rho_{1}$ is the principal part of $M(C)$. The observation now follows by induction.

To prove the lemma it suffices to consider an element $g \in J \cap F_{q}$ and representation $g=C_{r}^{e_{r}} \ldots C_{s}^{e_{s}} \bmod F_{q+1}$ where $C_{r}, \ldots, C_{s}$ are the basic commutators of weight $q$. The principal part of $M(g)$ is $\rho=e_{r} \rho_{r}+\cdots+e_{s} \rho_{s}$, where $\rho_{i}$ is the principal part of $M\left(C_{i}\right)$. Now one of the more important properties of basic commutators is that $\left\{\rho_{r}, \ldots, \rho_{s}\right\}$ is a linearly independent set [MKS]. Therefore, if $e_{i} \neq 0$ for some $C_{i}$ without repeats the $\rho$ contains terms in which no variable has total degree $>1$. On the other hand we can see that for any $g \in J$, every term of $M(g)-1$ must contains some variable with degree $>1 . J$ is the subgroup generated by all $\left[\bar{N}_{i}, \bar{N}_{i}\right]$ and if $g \in \bar{N}_{i}$, then every term of $M(g)-1$ contains $x_{i}$ with total degree $>0$. An easy calculation establishes the result.

4. We now show that the reduced structured group of a link is a homotopy invariant. In fact we will associate to a specific homotopy, in a functorial manner, an isomorphism (up to inner automorphisms) of the reduced structured groups which respects composition in the two contexts. Roughly speaking this can be done by identifying the reduced groups with the analogous group of the "trace" of the homotopy, but we will take a more combinatorial approach.

It is an easy consequence of general position that any homotopy can be approximated by a sequence of link isotopies and crossing changes (see Figure 1) which we explicitly describe as follows. Let $L$ be a link and $B$ a 3-ball in $S^{3}$ such that $B \cap L$ consists of two arcs from the same component of $L$ which is trivial in the sense that there is a diffeomorphism of $B$ onto the standard 3-ball which maps $L \cap B$ onto straight line segments. Let $L^{\prime}$ be another link satisfying:

(i) $L^{\prime} \cap\left(S^{3}-B\right)=L \cap\left(S^{3}-B\right)$.

(ii) $L^{\prime} \cap B$ is another pair of trivial arcs.

(iii) $L$ and $L^{\prime}$ have the same multiplicity and the order and orientation agree in (i).

We will say that $L$ and $L^{\prime}$ are related by an elementary homotopy in $B$. A homotopy will now denote a sequence of link isotopies and elementary homotopies.

Suppose we have an elementary homotopy between $L_{1}$ and $L_{2}$ in $B$. Let $G=$ $\pi_{1}\left(S^{3}-\left(B \cup L_{i}\right)\right)$. We use the same base-point for $G, G\left(L_{1}\right)$ and $G\left(L_{2}\right)$ and there are natural epimorphisms $\rho_{1}: G \rightarrow G\left(L_{i}\right)$. The kernel $R_{i}$ of $\rho_{i}$ can be described as follows. Choose meridians $\alpha_{1}, \alpha_{2} \in G$ for the two segments of one of the arcs emerging from $B$ and $\beta_{1}, \beta_{2}$ for the other arc. We may choose these elements to share the same stem (see Figure 2).

We have the relation $\alpha_{1} \beta_{1}=\beta_{2} \alpha_{2}$ in $G$, If we consider the corresponding elements in $G\left(L_{1}\right)$ or $G\left(L_{2}\right)$ we have the extra relation $\alpha_{1}=\alpha_{2}$ or $\beta_{1}=\beta_{2}$ depending on the position of the arcs in $B$. Thus $R_{i}$ is the normal closure of either $\alpha_{1} \alpha_{2}^{-1}$ or $\beta_{1} \beta_{2}^{-1}$.

We can define a reduced group $G$ from $G$ by dividing out the (normal) subgroup generated by all elements of the form $\left[\mu_{i}^{\prime}, \mu_{i}^{\prime \prime}\right]$ where $\mu_{i}^{\prime}, \mu_{i}^{\prime \prime}$ are meridians of the $i$ th component for an $i=1, \ldots, m$. Then $\rho_{i}$ induces epimorphisms $\rho_{i}: \mathcal{G} \rightarrow \mathcal{G}\left(L_{i}\right)$ whose kernel is the normal closure of $\alpha_{1} \alpha_{2}^{-1}$ or $\beta_{1} \beta_{2}^{-1}$. But since $\alpha_{i}$ and $\beta_{j}$ commute in $\mathcal{G}$ these are the same element and so we obtain an isomorphism $\rho_{2} \circ \rho_{1}^{-1}: \mathcal{G}\left(L_{1}\right) \rightarrow$ $\mathcal{G}\left(L_{2}\right)$. 


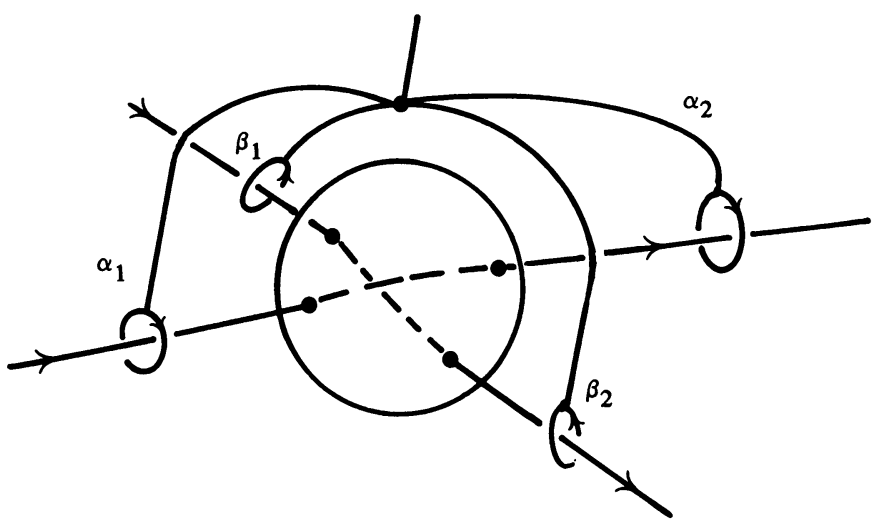

FIGURE 2

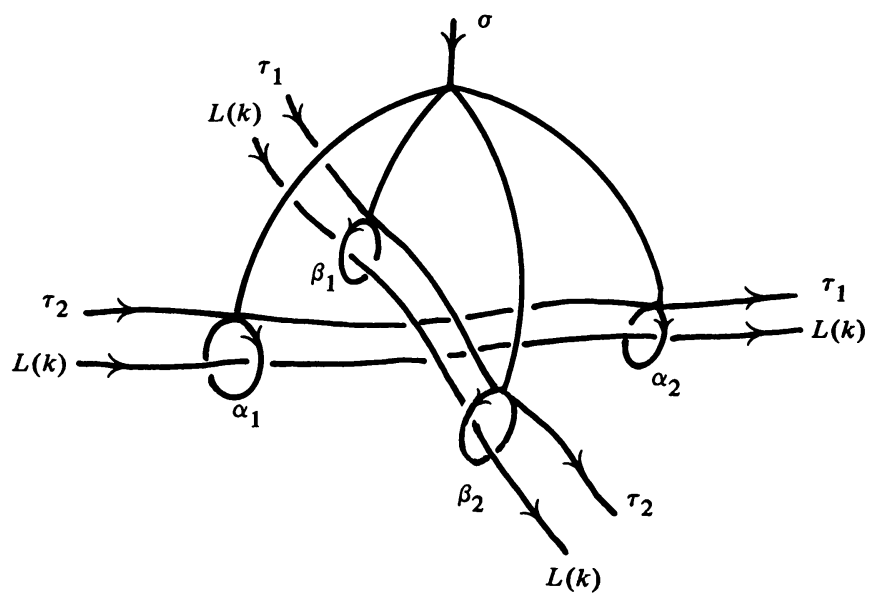

FIGURE 3

By taking compositions we have now defined a multiplicative transformation from homotopies of links to isomorphisms of reduced groups.

THEOREM 4. Suppose a homotopy between links $L$ and $L^{\prime}$ defines an isomorphism $\phi: \mathcal{G}(L) \rightarrow \mathcal{G}\left(L^{\prime}\right)$ and $\left\{\mu_{i}, \theta_{i}\right\}$ is a peripheral structure on $\mathcal{G}(L)$ (see $\S 2$ ).

Then there is a peripheral structure $\left\{\mu_{i}^{\prime}, \theta_{i}^{\prime}\right\}$ on $\mathcal{G}\left(L^{\prime}\right)$ such that $\mu_{i}^{\prime}=\phi\left(\mu_{i}\right)$ and $\theta_{i}^{\prime} \equiv \phi\left(\theta_{i}\right) \bmod N_{i}\left(L^{\prime}\right)$ for every $i$.

We will say that $\phi$ is a strong isomorphism between the structured reduced groups $\mathcal{G}(L)$ and $\mathcal{G}\left(L^{\prime}\right)$. An isomorphism $\phi$ which only has the property that $\phi\left(\mu_{i}\right)$ is an $i$-meridian, for every $i$, will be called special. Note that any special isomorphism $\phi$ satisfies $\phi N_{i}(L)=N_{i}\left(L^{\prime}\right)$ for every $i$.

PROOF OF THEOREM. We consider an elementary homotopy in a ball $B$ which changes a crossing of the $k$ th component. It is clear that we only have to worry about the $k$ th meridians and longitudes. Consider Figure 3. 


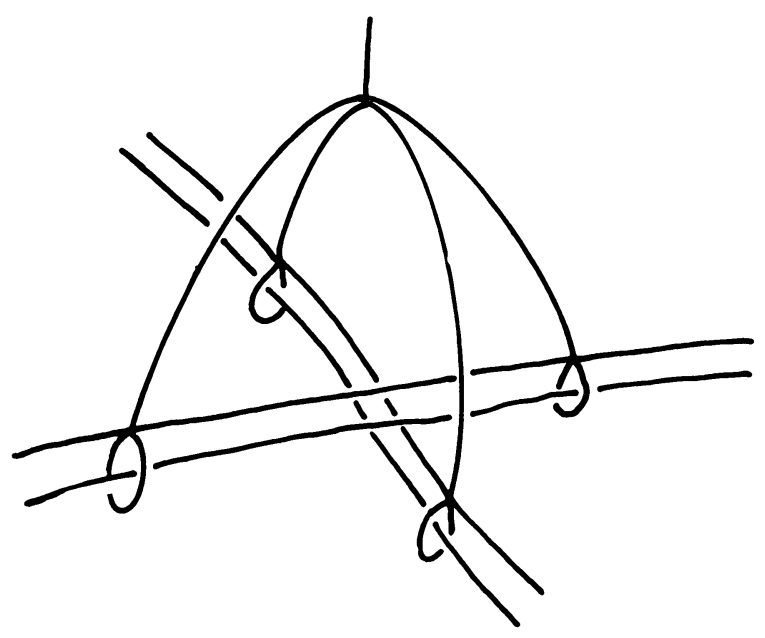

FIGURE 4

In this figure $\alpha_{1}, \alpha_{2}, \beta_{1}, \beta_{2}, \tau_{1}, \tau_{2}$ are closed paths in $S^{3}-(B \cap L)$ sharing the same stem $\sigma$. We also insist that the linking number of $\tau_{i}$ with $L(K)$ is zero. We may take $\mu_{k}$ to be represented by $\alpha_{1}$. Then $\lambda_{k}$ is represented by $\alpha_{1}^{-1} \beta_{2} \tau_{1} \tau_{2}$. We may draw a similar figure for $L^{\prime}$ near $B$ except that inside $B$ the crossings are changed (see Figure 4).

We take $\mu_{k}^{\prime}$ to be represented by $\alpha_{1}$ again, and now $\lambda_{k}^{\prime}$ is represented by $\alpha_{1} \tau_{1} \alpha_{1}^{-1} \tau_{2}$. The isomorphism $\mathcal{G}(L) \rightarrow \mathcal{G}\left(L^{\prime}\right)$ sends $\mu_{k}$ to $\mu_{k}^{\prime}$ and $\lambda_{k}$ to $\xi \lambda_{k}^{\prime}$ where $\xi$ is represented by $\left(\alpha_{1}^{-1} \beta_{2} \tau_{1} \tau_{2}\right)\left(\alpha_{1} \tau_{1} \alpha_{1}^{-1} \tau_{2}\right)^{-1}=\alpha_{1}^{-1} \beta_{2} \tau_{1} \alpha_{1} \tau_{1}^{-1} \alpha_{1}^{-1}$. We need to show that $\xi \in N_{k}\left(L^{\prime}\right)$. Note that $\beta_{2}=\alpha_{1} \beta_{1} \alpha_{1}^{-1}, \beta_{1}=\tau_{1}^{-1} \alpha_{2} \tau_{1}$ and $\alpha_{2}=\alpha_{1}$ in $G\left(L^{\prime}\right)$. Substituting for $\beta_{2}$ we find that $\xi$ can be represented by

$$
\alpha_{1}^{-1}\left(\alpha_{1} \tau_{1}^{-1} \alpha_{1} \tau_{1} \alpha_{1}^{-1}\right) \tau_{1} \alpha_{1} \tau_{1}^{-1} \alpha_{1}^{-1}=\tau_{1}^{-1} \alpha_{1} \tau_{1} \alpha_{1}^{-1} \tau_{1} \alpha_{1} \tau_{1}^{-1} \alpha_{1}^{-1}=\left[\tau_{1}^{-1},\left[\alpha_{1}, \tau_{1}\right]\right]
$$

which is conjugate to $\left[\tau_{1},\left[\tau_{1}, \alpha_{1}\right]\right]$, an element of $N_{k}\left(L^{\prime}\right)$. This completes the proof.

5. We now consider a single link $L$ and ask which automorphisms of the structured reduced group $\mathcal{G}(L)$ are induced by homotopies from $L$ to itself (self-homotopies of $L$ ). We call such automorphisms geometric. In addition to those induced by elementary homotopies, this will include those induced by ambient isotopies. It follows from the theorem of $\S 4$ that a geometric automorphism $\phi$ satisfies the following condition: For any peripheral structure $\left\{\mu_{i}, \theta_{i}\right\}$ of $\mathcal{G}(L)$, there exist elements $g_{i} \in \mathcal{G}(L)$ so that

(i) $\phi\left(\mu_{i}\right)=g_{i} \mu_{i} g_{i}^{-1}$

(ii) $\phi\left(\theta_{i}\right) \equiv g_{i} \theta_{i} g_{i}^{-1} \bmod N_{i}(L)$.

Automorphisms satisfying (i), (ii) will be called strong and those satisfying (i) special. We will say that the elements $\left\{g_{1}, \ldots, g_{m}\right\}$ of $\mathcal{G}(L)$ are admissible for $\phi$. A change of peripheral structure results in conjugation of the $\left\{g_{i}\right\}$. Note that a given strong automorphism and peripheral structure may have many admissible sets and not every set is admissible. 
The question of which automorphisms of structured reduced groups are geometric is related to the homotopy classification of links by the following theorem which is a simple generalization of [ $\mathbf{M}$, Theorem 3].

THEOREM 5. Let $L$ be a link and $l_{1}, l_{2}$ oriented simple closed curves in the complement of $L$. Let $L_{i}$ be the link obtained by adjoining $l_{1}$ to $L(i=1,2)$. Suppose $l_{i}$ represents $\alpha_{i} \in \mathcal{G}(L)$-up to conjugacy. Then $L_{1}$ is homotopic to $L_{2}$ if and only if there exists a geometric automorphism $\phi$ of $\mathcal{G}(L)$ such that $\phi\left(\alpha_{1}\right)=\alpha_{2}$.

Note that every inner automorphism of $\mathcal{G}(L)$ is geometric. The isotopy which moves the basepoint about a closed curve representing $g \in G(L)$ but leaves $L$ fixed will induce the inner automorphism of $G(L)$ defined by conjugating with $g$.

Milnor's Theorem shows that $L_{1}$ is homotopic to $L_{2}$ if $\alpha_{1}=\alpha_{2}$. The point of this generalization is that we obtain necessary and sufficient conditions by bringing in automorphisms of $\mathcal{G}(L)$. The proof is an easy consequence of Milnor's Theorem.

If $L_{1}$ is homotopic to $L_{2}$, we can restrict the homotopy to $L$ to obtain a selfhomotopy. The induced automorphism of $\mathcal{G}(L)$ will clearly do. Conversely, given $\phi$ induced by a self-homotopy of $L$, we use the following lemma to extend this to a homotopy from $L_{1}$ to some link $L_{3}$. Obviously $L_{3}$ consists of $L$ together with an extra component $l_{3}$-if $l_{3}$ represents $\alpha_{3} \in \mathcal{G}(L)$ then it is clear that $\phi\left(\alpha_{1}\right)=\alpha_{3}$. We now compare $L_{2}$ and $L_{3}$ : They both contain $L$ as principal sublink and the extra components represent the same element of $\mathcal{G}(L)$. Thus we can apply Milnor's Theorem to conclude that $L_{2}$ and $L_{3}$ are homotopic. This completes the proof.

LEMMA 5. Suppose $L_{1}$ is a link with sublink $K_{1}$ and a given homotopy of $K_{1}$ with $K_{2}$. Then the homotopy extends to a homotopy of $L_{1}$ with some link $L_{2}$ containing $K_{2}$ as a sublink.

Since isotopies obviously satisfy this extension property we only need consider elementary homotopies. If $B$ is the ball in which an elementary homotopy of $K_{1}$ occurs then we need only check that $\left(L_{1}-K_{1}\right) \cap B$ can be isotopically deformed out of $B$ rel $K_{1}$. We leave this as an exercise for the reader.

It seems much more difficult to decide whether this lemma is true in higher dimensions. For example, it would imply that any imbedding $S^{p}+S^{q} \subseteq S^{m}$ is null-homotopic if $\pi_{p}\left(S^{m-q-1}\right)=0$-when $p=q=2, m=4$ a proof has been announced ([HS] - also see [MR, FR]). In the context of link maps, i.e. maps of several spheres into $S^{m}$ allowing self-intersections in each component but no intersections between different components, this lemma is false. For example, there are nontrivial link maps $S^{2}+S^{2} \rightarrow S^{4}$ (see [FR]) but, as mentioned above, this lemma would preclude such a possibility.

By this theorem we have, in some sense, reinterpreted the homotopy classification question as the problem of determining which automorphisms of the reduced structured group are geometric. We can, in fact, relate this problem to the specific question of whether the reduced structured group determines the homotopy class of a link.

COROLLARY 5. Let $L$ be a link and suppose that the sublinks $L_{r}=\{L(1)$, $L(2), \ldots, L(r)\}, 2 \leq r<m$, have the property that every strong automrophism of $\mathcal{G}\left(L_{r}\right)$ is geometric. Then any link whose reduced structured group is strongly isomorphic to $\mathcal{G}(L)$ is homotopic to $L$. 
PROOF. We begin an induction on the number of components of $L$ by noticing that all knots are homotopic. Now suppose $L^{\prime}$ is a link and $\Phi: \mathcal{G}(L) \rightarrow \mathcal{G}\left(L^{\prime}\right)$ is a strong isomrophism. If $K$ and $K^{\prime}$ are the two principal sublinks we have an induced strong isomorphism $\phi: \mathcal{G}(K) \approx \mathcal{G}\left(K^{\prime}\right)$, since $\Phi$ identifies meridians of the last components. By induction we can assume that $K$ and $K^{\prime}$ are homotopic, and, therefore, by Lemma 5 we may even assume $K=K^{\prime}$. We can now regard $\phi$ as a strong automorphism of $\mathcal{G}(K)$ and, by property (ii) applied to $\Phi$, we see that $\phi$ identifies the classes represented by the remaining components. By induction we may conclude that $\phi$ is geometric and so apply Theorem 5 to conclude $L$ and $L^{\prime}$ are homotopic.

6. We use Theorem 5 to formulate a practical recursive approach to the homotopy classification of links. Suppose $K$ is a fixed $m$-component link with a peripheral structure and we consider the class of links obtained by adjoining one more component to $K$. We will use the class of this component in $\mathcal{G}(K)$ to define a set of numerical invariants. The indeterminacy of these invariants arises from the relations in and the automorphisms of $\mathcal{G}(K)$. By just considering geometric automorphisms, the indeterminacy is precisely that needed for homotopy classification. If we consider strong automorphisms, then the indeterminacy may be too large if it turns out that there are automorphisms which are not geometric.

To begin will need a version of the Hall Basis Theorem in the context of the reduced free group $\mathcal{F}$ defined to be the quotient $F / J$ where $F$ is the free group with basis $\left\{\mu_{i}\right\}$ (see $\S 3$ ). Note that $\mathcal{F}$ is the reduced group of the trivial link, where a peripheral structure $\left\{\mu_{i}, \theta_{i}\right\}$ is given by: $\theta_{i} \equiv 1$. We will say that $C_{1}, C_{2}, \ldots$ is a set of reduced basic commutators if it is obtained from a set of basic commutators by deleting those with repeats. Note that any set of reduced basic commutators is finite. The following proposition is a direct consequence of the Hall Basis Theorem and Lemma 3.

PROPOSITION 6. If $C_{1}, \ldots, C_{n}$ is a set of reduced basic commutators in $\left\{\mu_{i}\right\}$, then every element of $\mathcal{F}$ has a unique representation in the form $C_{1}^{e_{1}} C_{2}^{e_{2}} \cdots C_{n}^{e_{n}}$.

Now suppose $L$ is a link whose principal sublink is $K$, and the last component of $L$ defines the element $\alpha \in \mathcal{G}(K)$-up to conjugacy. The peripheral structure on $K$ determines generators $\left\{\mu_{1}\right\}$ of $\mathcal{G}(K)$. We write $\alpha=C_{1}^{e_{1}} \cdots C_{n}^{e_{n}}$ and refer to $\left\{e_{1}, \ldots, e_{n}\right\}$ as the commutator numbers of $L$. Since the peripheral structure on $K$ and the set of reduced basic commutators are prescribed the only source of indeterminacy in the commutator numbers are the relations in $\mathcal{G}(K)$ and, by Theorem 5, the geometric automorphisms of $\S 5$.

More specifically we have, from Proposition 3 an isomorphism $\mathcal{G}(K) \approx \mathcal{F} / \mathcal{N}$, where $\mathcal{N}$ is the normal closure of elements $\left\{\left[\mu_{i}, \bar{\lambda}_{i}\right]\right\}$ determined by the peripheral structure $\left\{\mu_{i}, \lambda_{i}\right\}$ and a prescribed choice of elements $\bar{\lambda}_{i}$ in $\mathcal{F}$ representing $\lambda_{i} \in$ $\mathcal{G}(K)$. We also fix a set of generators $\left\{\phi_{i}\right\}$ of the group of geometric automorphisms of $\mathcal{G}(K)$ and, for each $\phi_{i}$ a set of elements $\left\{g_{i j}\right\}$ in $\mathcal{F}$ whose reductions to $\mathcal{G}(K)$ are admissible for $\phi_{i}$. From the $\left\{\bar{\lambda}_{i}\right\}$ and $\left\{g_{i j}\right\}$ we define a set of transformations of the commutator number $\left\{e_{i}\right\} \mapsto\left\{e_{i}^{\prime}\right\}$ :

(i) For each $\bar{\lambda}_{i}$, define $\left\{e_{j}^{\prime}\right\}$ by the formula

$$
C_{1}^{e_{1}} \cdots C_{n}^{e_{n}} \cdot\left[\bar{\lambda}_{i}, \mu_{i}\right]=C_{1}^{e_{1}^{\prime}} \cdots C_{n}^{e_{n}^{\prime}} .
$$


(ii) For each $g_{i 1}, \ldots, g_{i m}$, define $\left\{e_{j}^{\prime}\right\}$ by the formula

$$
\bar{C}_{1}^{e_{1}} \cdots \bar{C}_{n}^{e_{n}}=C_{1}^{e_{1}^{\prime}} \cdots C_{n}^{e_{n}^{\prime}}
$$

where $C_{r} \mapsto \bar{C}_{r}$ under the substitution of $g_{i j} \mu_{j} g_{i j}^{-1}$ for $\mu_{j}(j=1, \ldots, m)$.

We will refer to the transformations in (i) as $R$-transformations and those in (ii) as A-transformations. We may, in fact, ignore one of the relations and so obtain $(m-1) R$-transformations. If we consider strong automorphisms instead of geometric automorphisms in (ii), we will refer to them as $A^{\prime}$-transformations. The equivalence relation among commutator numbers generated by $R$ and $A$ (resp. $A^{\prime}$ ) transformations will be referred to as $\rho$-equivalence (resp. $\rho^{\prime}$-equivalence). Of course $\rho$-equivalence is finer than $\rho^{\prime}$-equivalence.

THEOREM 6. Given $K$ with a prescribed peripheral structure and elements $\left\{\bar{\lambda}_{i}\right\}$ and $\left\{g_{i j}\right\}$ in $\mathcal{F}$, as described above, then the $\rho$-equivalence class (and so the $\rho^{\prime}$ equivalence class) of the commutator numbers of any link $L$ with principal sublink $K$ is a homotopy invariant of $L$. If two such links have $\rho$-equivalent commutator numbers then they are homotopic.

In light of Theorem 5, we only have to check that two elements of $\mathcal{G}(K)$ are conjugate up to geometric (strong) automorphisms if and only if their commutator numbers are $\rho$-equivalent $\left(\rho^{\prime}\right.$-equivalent). First of all it is clear that two elements of $\mathcal{G}(K)$ admit commutator numbers that are equal under a sequence of $A$-transformations ( $A^{\prime}$-transformations) and their inverses if and only if there is a geometric (strong) automorphism identifying them. It is also easy to see that two sets of commutator numbers determine the same element of $\mathcal{G}(K)$ if and only if they are related by a sequence of transformation similar to $R$-transformations except that we must consider the more general case of conjugates $g\left[\bar{\lambda}_{i}, \mu_{i}\right] g^{-1}$ in (i), $g \in \mathcal{F}$. But since all inner automorphisms are geometric we may express such a transformation as a sequence of $R$ and $A\left(A^{\prime}\right)$ transformations. This completes the proof.

\section{PART II}

7. We begin with a technique for constructing geometric automorphisms.

THEOREM 7. Suppose $L$ is an m-component link whose kth component $L(k)$ bounds a disk $\Delta$ in $S^{3}$ whose interior intersects $L$ only at points of the rth component $L(r)(1 \leq k, r \leq m)$. Suppose $\left\{\mu_{i}\right\}$ is a set of meridians for $L$ that have representative curves whose stems are all disjoint from $\Delta$. Then a geometric automorphism $\phi$ of $\mathcal{G}(L)$ is defined by: $\mu_{k} \mapsto \mu_{r} \mu_{k} \mu_{r}^{-1}, \mu_{i} \mapsto \mu_{i}$ for $i \neq k$. If the interior of $\Delta$ is disjoint from $L$ entirely then $\phi$ is induced by an isotopy of $L$.

Proof. Choose an arc $\tau$ from a point of $L(r)$ to a point of $L(k)$ which is otherwise disjoint from $L, \Delta$ and the representatives of $\left\{\mu_{i}\right\}$. The homotopy, which will move only $L(r)$, consists of pushing a segment of $L(r)$ along $\tau$, sliding it under $\Delta$, back over $\Delta$ and finally pulling it back along $\tau$. See Figures 5 and 6 .

The intersections that occur during this deformation occur between $L(r)$ and the intersections of $L$ with $\stackrel{\circ}{\Delta}$. The assumption of the theorem implies that we have a homotopy or, in the case where $\delta \cap L$ is empty, an isotopy. 


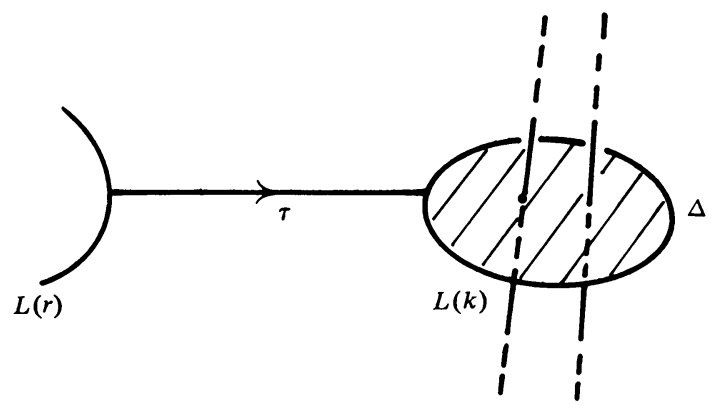

FIGURE 5
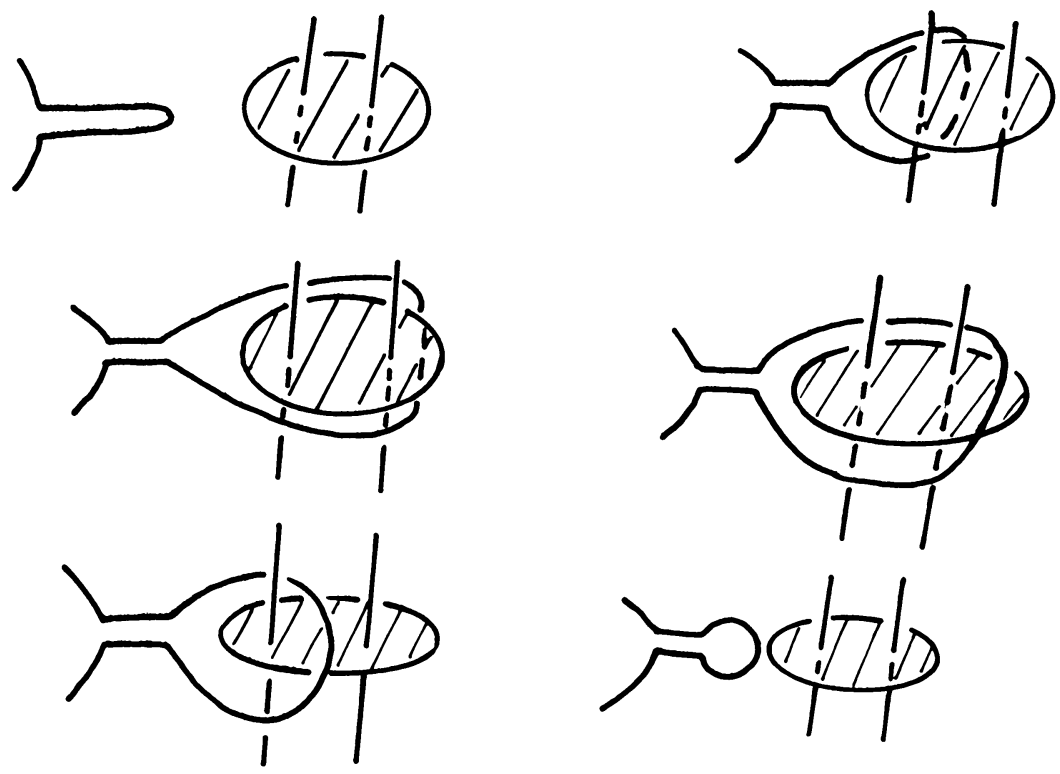

\section{FIGURE 6}

The automorphism $\phi$ of $\mathcal{G}(L)$ induced by this homotopy does not alter $\mu_{i}$ if $i \neq k$, since they can be represented by curves disjoint from $\Delta$ and $\tau$. Now suppose $\gamma_{i}, m_{i}$ are the curves defined in $\S 1$, so that $\gamma_{i} m_{i} \gamma_{i}^{-1}$ represents $\mu_{i}$. Then it is easy to see that $\phi\left(\mu_{k}\right)$ is represented by the curve $\left(\gamma_{k} \tau^{-1} m_{r} \tau\right) m_{k}\left(\tau^{-1} m_{\gamma}^{-1} \tau \gamma_{k}^{-1}\right)$. See Figure 7. If we choose $\tau=\gamma_{r}^{-1} \gamma_{k}$, then $\phi\left(\mu_{k}\right)=\mu_{r} \mu_{k} \mu_{r}^{-1}$.

8. As our first application we determine the geometric automorphisms of almost trivial links. Recall from [M] that a link is almost trivial if any proper sublink is homotopic to the trivial link.

THEOREM 8. If $L$ is an $m$-component almost trivial link, then every special automorphism is geometric. Furthermore every set of elements $g_{1}, \ldots, g_{m}$ of $\mathcal{G}(L)$ is admissible for a special automorphism. If $L$ is trivial then every special automorphism is induced by an isotopy of $L$. 


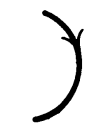

$L(r)$

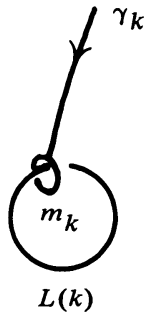

$L(k)$

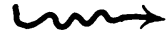

$L(r)$

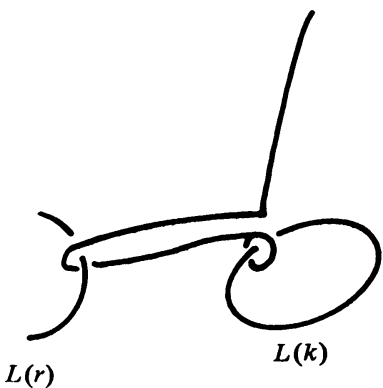

FIGURE 7

We first deal with the case of $L$ trivial. Then $\mathcal{G}(L) \approx \mathcal{F}$ and a peripheral structure $\left\{\mu_{i}, \theta_{i}\right\}$ is given by $\theta_{i}=1$ and $\left\{\mu_{i}\right\}$ the reduction of a basis of $F$. For any $1 \leq r, s \leq m$ let $\phi_{r, s}$ be the automorphism of $\mathcal{F}$ defined by

$$
\phi_{r, s}\left(\mu_{i}\right)= \begin{cases}\mu_{s} \mu_{r} \mu_{s}^{-1}, & i=r, \\ \mu_{i}, & i \neq r .\end{cases}
$$

In fact $\phi_{r, s}$ is induced from an automorphism of $F$ defined by the same formula. It is known that these elements generate the group of special automorphisms (automorphisms which take every every basis element to some conjugate) of $F$ (see $[\mathbf{H}, \mathbf{K o}])$. We will need

LEMMA 8. Every set of elements $g_{1}, \ldots, g_{m}$ of $\mathcal{F}$ is admissible for some special automorphism of $\mathcal{F}$. Furthermore, $\left\{\phi_{r, s}\right\}$ are a set of generators of this group of special automorphisms.

PROOF. We first point out that for any special automorphism of $₹$ there exists an admissible set $g_{1}, \ldots, g_{m}$ such that each $g_{j}$ can be written as a word in $\left\{\mu_{i}\right\}$ which does not contain $\mu_{j}$. In fact if we have $g_{j}=v_{j} \mu_{j}^{e} w_{j}$, then

$$
g_{j} \mu_{j} g_{j}^{-1}=v_{j} \mu_{j}^{e}\left(w_{j} \mu_{j} w_{j}^{-1}\right) \mu_{j}^{-e} v_{j}^{-1}=v_{j} w_{j} \mu_{j} w_{j}^{-1} v_{j}^{-1}
$$

since $\mu_{j}$ commutes with $w_{j} \mu_{j} w_{j}^{-1}$ in $\mathcal{F}$. Therefore $g_{j}$ may be replaced by $v_{j} w_{j}$. Iterating this process will remove all appearances of $\mu_{j}$ in $g_{j}$.

Now suppose $g_{1}, \ldots, g_{m}$ is any set of elements of $\mathcal{F}$ with the property that $\mu_{j}$ does not appear in $g_{j}$. Suppose, for some $1 \leq k \leq m$, that $g_{i}=1$ if $i>k$. We will show, by induction on $k$, that the endomorphism $\phi$ of $₹$ defined by $\phi\left(\mu_{i}\right)=g_{i} \mu_{i} g_{i}^{-1}$ is an automorphism and can be written as a composition of $\left\{\phi_{r, s}^{ \pm 1}: 1 \leq r, s \leq m\right\}$. Consider the endomorphism $\psi$ of $\mp$ defined by

$$
\psi\left(\mu_{i}\right)= \begin{cases}g_{k} \mu_{k} g_{k}^{-1}, & i=k, \\ \mu_{i}, & i \neq k .\end{cases}
$$

Since $g_{k}$ does not involve $\mu_{k}$, it is clear that $\psi$ is a product of $\left\{\phi_{k s}^{ \pm 1}: 1 \leq s \leq m\right\}$ and so an automorphism. If $\sigma=\psi^{-1} \circ \phi$ then $\sigma\left(\mu_{i}\right)$ is a conjugate of $\mu_{i}$, for every $i$, and $\sigma\left(\mu_{i}\right)=\mu_{i}$ if $i \geq k$. By induction we have that $\sigma$ is an automorphism and a product of $\left\{\phi_{r, s}: 1 \leq r, s \leq m\right\}$. We then have the conclusion for $\phi=\psi \circ \sigma$.

This proves the lemma. 

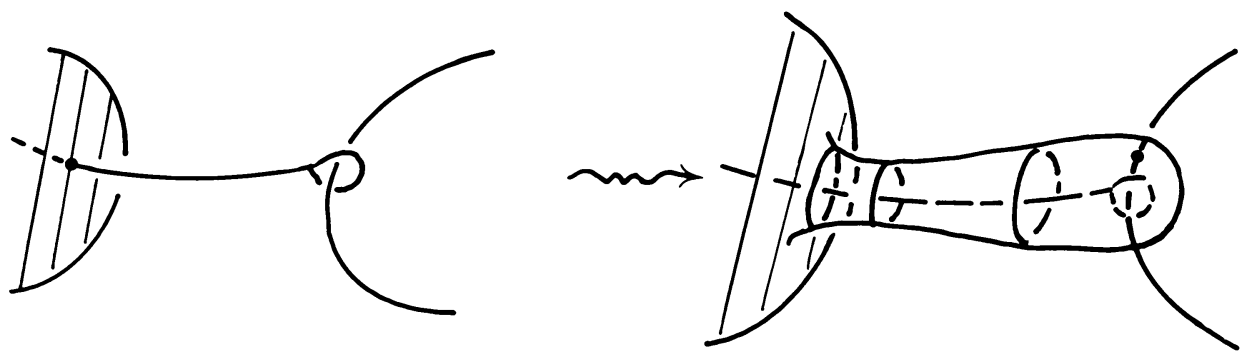

FIGURE 8

Now suppose $L$ is the trivial link. We can choose disjoint disks $\Delta_{1}, \ldots, \Delta_{m}$ in $S^{3}$ whose boundaries form $L$. Choose a set of meridians for $L$ whose stems are disjoint from $\left\{\Delta_{i}\right\}$. Then it follows from Theorem 7 that every $\phi_{r, s}$ is geometric and, in fact, induced by an isotopy of $L$. By Lemma 8 this is also true for every special automorphism.

We denote the following consequence of the above argument. Given any set of meridians $\left\{\mu_{i}\right\}$ in $\mathcal{G}(L)$, there exist disjoint disks $\left\{\Delta_{i}\right\}$ in $S^{3}$ whose boundaries form $L$ such that $\left\{\mu_{i}\right\}$ are presented by curves with stems disjoint from $\left\{\Delta_{i}\right\}$-we will say that $\left\{\Delta_{i}\right\}$ are in good position for $\left\{\mu_{i}\right\}$. In fact, choose any disks $\left\{\Delta_{i}^{\prime}\right\}$ and meridians $\left\{\mu_{i}^{\prime}\right\}$ such that $\left\{\Delta_{i}^{\prime}\right\}$ are in good position for $\left\{\mu_{i}^{\prime}\right\}$. By the lemma, theie is a special automorphism $\phi$ of $\mathcal{G}(L)$ such that $\phi\left(\mu_{i}^{\prime}\right)=\mu_{i}$ and by the argument above $\phi$ is induced by an isotopy of $L$. This isotopy will transform $\left\{\Delta_{i}^{\prime}\right\}$ into the desired $\left\{\Delta_{i}\right\}$.

We now take $L$ to be an almost trivial link. Let $\left\{\mu_{i}\right\}$ be any set of meridians of $L$, which defines an epimorphism $\mathcal{F} \rightarrow \mathcal{F}\left(L^{\prime}\right)$ by Proposition 3. We will show that every automorphism $\phi_{r, s}$ of $\mathcal{F}$ induces an automorphism of $\mathcal{G}\left(L^{\prime}\right)$ which is geometric. By Lemma 8 this will prove the theorem.

Let $L^{\prime}$ be the sublink of $L$ obtained by removing the $r$ th component. Then $L^{\prime}$ is homotopic to the trivial link and, by Lemma 5 , we can assume $L^{\prime}$ is trivial. We may choose disjoint imbedded disks $\Delta_{1}, \ldots, \hat{\Delta}_{r}, \ldots, \Delta_{m}$ in good position for the meridians of $L^{\prime}$ induced by $\left\{\mu_{i}\right\}$. We would like to apply Theorem 7 to obtain the desired geometric automorphism. The only problem is that the stem of the curve representing $\mu_{r}$ might intersect $\Delta_{s}$. But these intersections may be eliminated, at the cost of introducing more intersections of $\Delta_{s}$ with $L(r)$, by pushing a little disk of $\Delta_{s}$ at an intersection point along the stem past the meridian circle (see Figure 8).

Since the stem does not intersect any other $\Delta_{i}, \Delta_{s}$ will remain disjoint from every other $\Delta_{i}$.

This completes the proof of the theorem.

COROLlaRY 8. Suppose $L$ is an m-component link such that one if its $(m-1)$ component sublinks is almost trivial. Then any other link $L^{\prime}$ is homotopic to $L$ if and only if $\mathcal{G}(L)$ is strongly isomorphic to $\mathcal{G}\left(L^{\prime}\right)$.

This is an immediate consequence of Corollary 5 together with the theorem above. In fact, by examining the proof of Corollary 5 we only need require the 
isomorphism $\mathcal{G}(L) \approx \mathcal{G}\left(L^{\prime}\right)$ to be special and preserve the preperipheral structure (see $\S 2$ ).

This corollary applies to all 3-component links and to 4-component links whose linking numbers $l_{i j}, 1 \leq i, j \leq 4$, satisfy the following property.

For some $k, l_{i j}=0$ unless $i=k$ or $j=k$.

\section{PART III}

9. The rest of this paper will be devoted to determining the geometric automorphisms of links with 3 or fewer components. As a consequence we will be able to give an explicit homotopy classification of links with 4 or fewer components in terms of the commutator numbers defined in $\S 6$. For 3-component links such a classification already appears in $[\mathbf{M}]$, but we will go through this case as a warm-up for the considerably more complicated 4-component case.

THEOREM 9. If $L$ is a link with 3 or fewer components, then every strong automorphism of $\mathcal{G}(L)$ is geometric. The homotopy class of any link with 4 or fewer components is determined by the strong isomorphism class of its reduced structured group.

The second assertion follows from the first and Corollary 5 .

The case of one component is trivial. It has pointed out many times in the literature that the linking number is a complete homotopy invariant for 2-component links (see e.g. [M]).

Suppose $L$ is a 2-component link. Since we may replace $L$ by any other link in its homotopy class, we may assume $L$ is a twist link with $k$ twists, where $k$ is the linking number. A standard computation shows that $L$ has a peripheral structure $\left\{\mu_{i}, \lambda_{i}\right\}$ where $\lambda_{1}=\mu_{2}^{k}, \lambda_{2}=\mu_{1}^{k}$. Since any special automorphism of $\mathcal{F}$ is easily seen to be inner, this is also true for $\mathcal{G}(L)$ and so they are all geometric.

We now show to use this fact to classify 3-component links by explicitly describing the indeterminacy (i.e. $\rho$-equivalence) of the commutator numbers. We shall write $x=\mu_{1}, y=\mu_{2}$. A set of reduced basic commutators of $\{x, y\}$ is $\{x, y[x, y]\}$.

If $L$ is a 3-component link with principal sublink $K$, then the linking number $k$ of $K$ is the first invariant of $L$ to record. The commutator numbers $\left\{e_{1}, e_{2}, e_{3}\right\}$ of $L$ are the exponents in the representation $\alpha=x^{e_{1}} y^{e_{2}}[x, y]^{e_{3}}$ where $\alpha \in \mathcal{G}(K)$ is (up to conjugacy) the class represented by the third component of $L$ in $\mathcal{G}(K)$ and $x, y$ are the meridian generators of $\mathcal{G}(K)$ corresponding to a prescribed peripheral structure.

The $R$-transformation is determined by rewriting $\alpha\left[\mu_{i}, \lambda_{i}\right](i=1$ or 2$)$ in the standard form

$$
\alpha\left[x, y^{k}\right]=x^{e_{1}} y^{e_{2}}[x, y]^{e_{3}}[x, y]^{k}=x^{e_{1}} y^{e_{2}}[x, y]^{e_{3}+k} .
$$

The $A$-transformations are determined by admissible sets for generators of the group of geometric (=inner) automorphisms. We may take $(1, x)$ and $(y, 1)$, corresponding to conjugation by $x$ and $y$, respectively. We then compute

$$
\begin{gathered}
x^{e_{1}}\left(x y x^{-1}\right)^{e_{2}}\left[x, x y x^{-1}\right]^{e_{3}}=x^{e_{1}}[x, y]^{e_{2}} y^{e_{2}}[x, y]^{e_{3}} \\
=x^{e_{1}} y^{e_{2}}[x, y]^{e_{2}+e_{3}} \\
\left(y x y^{-1}\right)^{e_{1}} y^{e_{2}}\left[y x y^{-1}, y\right]^{e_{3}}=x^{e_{1}} y^{e_{2}}[y, x]^{e_{1}} y^{e_{2}}[y, x]^{e_{1}}[x, y]^{e_{3}} \\
=x^{e_{1}} y^{e_{2}}[x, y]^{e_{3}-e_{1}} .
\end{gathered}
$$


We see that $e_{1}, e_{2}$ are unchanged by $R$ or $A$-transformations. This is a general fact - the exponents of the meridian generators will be invariant under $\rho$ equivalence. In fact they are just the linking numbers of the last component of $L$ with the other components.

Finally we see that $e_{3}$ can be changed by any multiple of $\left(k, e_{1}, e_{2}\right)$. Thus we conclude that a complete set of homotopy invariants for $L$ is given by the three linking numbers $k_{1}, k_{2}, k_{3}$ and $d=e_{3} \bmod \left(k_{1}, k_{2}, k_{3}\right)$, which coincides with $\bar{\mu}_{123}$ (see $[\mathbf{M}])$.

10. We now take up the task of determining the geometric automorphisms of 3-component links. At the same time we will discuss the related classification of 4-component links. In fact, the interplay between these two problems will be an indispensable part of the proof of Theorem 9.

As a first step we describe $\rho$-equivalence when $K$ is the trivial link. In other words we consider $\mathcal{F}$ and record the $A$-transformations corresponding to the generators $\left\{\phi_{r, s}\right\}$ of the group of special automorphisms of $\mathcal{F}$.

We write $x=\mu_{1}, y=\mu_{2}, z=\mu_{3}$ and the set of reduced basic commutators $x, y, z,[x, y],[x, z],[y, z],[y,[x, z]],[z,[x, y]]$. If $\alpha \in \mathcal{F}$, then we write

$$
\alpha=x^{e_{1}} y^{e_{2}} z^{e_{3}}[x, y]^{e_{4}}[x, z]^{e_{5}}[y, z]^{e_{6}}[y,[x, z]]^{e_{7}}[z,[x, y]]^{e_{8}}
$$

to determine the commutator numbers $\left\{e_{i}: 1 \leq l i \leq 8\right\}$ of $\alpha$. The $A$-transformations are obtained by writing $\phi_{r, s}(\alpha)$ in this form. We omit the details of this computation, which is straightforward, and record them in Table 1.

TABLE 1

\begin{tabular}{cccccc} 
& 4 & 5 & 6 & 7 & 8 \\
\hline$(1,2)$ & $-e_{1}$ & 0 & 0 & 0 & $e_{1} e_{3}+e_{5}$ \\
$(1,3)$ & 0 & $-e_{1}$ & 0 & $e_{1} e_{2}+e_{4}$ & 0 \\
$(2,1)$ & $e_{2}$ & 0 & 0 & 0 & $-e_{2} e_{3}-e_{6}$ \\
$(2,3)$ & 0 & 0 & $-e_{2}$ & $-e_{4}$ & $e_{4}$ \\
$(3,1)$ & 0 & $e_{3}$ & 0 & $e_{6}$ & 0 \\
$(3,2)$ & 0 & 0 & $e_{3}$ & $e_{5}$ & $e_{5}$
\end{tabular}

The entry in row $(r, s)$ and column $t$ represents the difference $e_{t}^{\prime}-e_{t}$ where $\left\{e_{t}\right\} \mapsto\left\{e_{t}^{\prime}\right\}$ is the $A$-transformation defined by $\phi_{r, s}$. Note that $t=1,2,3$ are omitted from the table since they are unchanged by any $A$-transformation.

It will be usefull to note that the subgroup of inner automorphisms of $\mathcal{F}$ is generated by $\phi_{21} \circ \phi_{31}, \phi_{12} \circ \phi_{32}$ and $\phi_{13} \circ \phi_{23}$ (conjugation by $x, y, z$, respectively) and the quotient group of outer special automorphisms of $\mathcal{F}$ is free abelian with basis $\phi_{12}, \phi_{21}$ and $\phi_{13}$. We will write $\Phi(a, b, c)=\phi_{13}^{a} \circ \phi_{12}^{b} \circ \phi_{21}^{c}$. The commutators $\left[\phi_{i j}, \phi_{r s}\right]$ do not affect $e_{i}$ for $i \leq 6$.

We may simplify Table 1 by replacing some of the $\phi_{r s}$ by other generators:

$$
\phi_{12}^{\prime}=\phi_{12} \circ\left[\phi_{31}, \phi_{12}\right]^{e_{1}}, \phi_{13}^{\prime}=\phi_{13} \circ\left[\phi_{21}, \phi_{13}\right]^{-e_{1}}, \phi_{21}^{\prime}=\phi_{21} \circ\left[\phi_{12}, \phi_{31}\right]^{e_{2}} \text {. }
$$

That these are also generators depends on the fact that any commutator of weight 3 in the $\left\{\phi_{r s}\right\}$ is trivial. Table 2 is the simplified table of $A$-transformations. 


\section{TABLE 2}

\begin{tabular}{cccccc} 
& 4 & 5 & 6 & 7 & 8 \\
\hline$(1,2)^{\prime}$ & $-e_{1}$ & 0 & 0 & 0 & $e_{5}$ \\
$(1,3)^{\prime}$ & 0 & $-e_{1}$ & 0 & $e_{4}$ & 0 \\
$(2,1)^{\prime}$ & $e_{2}$ & 0 & 0 & 0 & $-e_{6}$ \\
$(2,3)$ & 0 & 0 & $-e_{2}$ & $-e_{4}$ & $e_{4}$ \\
$(3,1)$ & 0 & $e_{3}$ & 0 & $e_{6}$ & 0 \\
$(3,2)$ & 0 & 0 & $e_{3}$ & $e_{5}$ & $e_{5}$
\end{tabular}

11. We now consider an arbitrary 3-component link. By the homotopy classfication of $\S 9$ we may take $K$ to be the link $L(k, l, r ; d)$ illustrated in Figure 9.

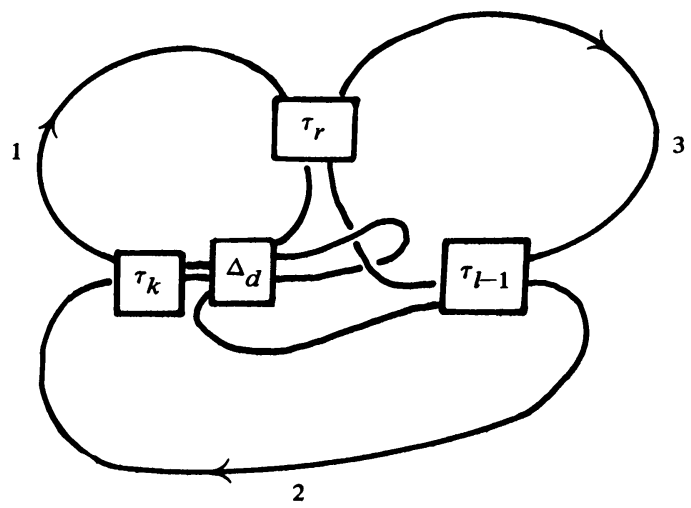

FIGURE 9

The components are numbered and oriented and the peripheral structure is determined by choosing stems connecting the dots on the link components to a basepoint above the page. The boxes enclose Skein elements illustrated in Figure 10.

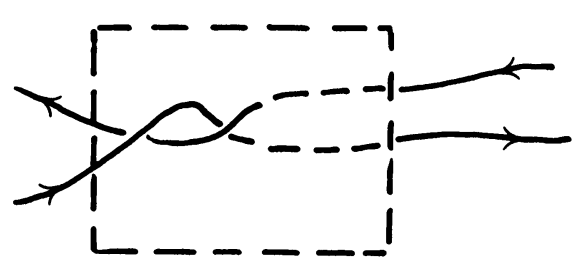

$\tau_{k}: k$ full twists

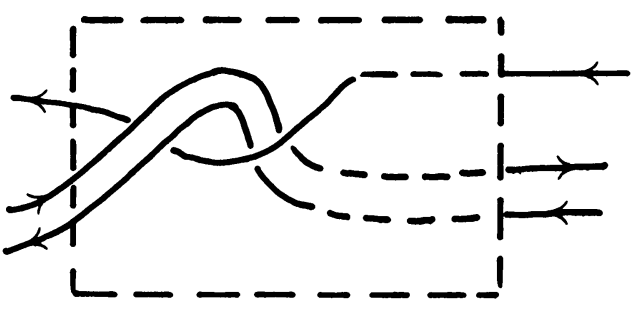

$\Delta_{d}: d$ full twists

FIGURE 10

The linking numbers of the three components are $k, l, r$ and $d$ is the invariant of §. A straightforward computation shows that, for the given peripheral structure, 
the longitudes in $\mathcal{G}(K)$ can be expressed as follows:

$$
\begin{aligned}
& \lambda_{1}=y^{K} z^{r}[x, y]^{\left(\begin{array}{c}
K \\
2
\end{array}\right)}[x, y]^{\left(\begin{array}{c}
r \\
2
\end{array}\right)}[y, z]^{d}, \\
& \lambda_{2}=x^{K} z^{l}[x, y]^{\left(\begin{array}{c}
K \\
2
\end{array}\right)}[z, x]^{d}[y, z]^{\left(\begin{array}{c}
l \\
2
\end{array}\right),} \\
& \lambda_{3}=x^{r} y^{l}[x, y]^{d}[x, z]^{\left(\begin{array}{c}
r \\
2
\end{array}\right)}[y, z]^{\left(\begin{array}{l}
l \\
2
\end{array}\right),}
\end{aligned}
$$

modulo commutators of weight 3 . Therefore, the relations for $\mathcal{G}(K)$ in the presentation given by Proposition 3 are

$$
\begin{aligned}
& {[x, y]^{k}[x, z]^{z}[x,[y, z]]^{d}=1,} \\
& {[y, x]^{k}[y, z]^{l}[y,[z, x]]^{d}=1,}
\end{aligned}
$$

We can ignore the third relation since it is a consequence of these two.

To determine the special automorphisms of $\mathcal{G}(K)$ we can consider the automorphisms $\Phi=\Phi(a, b, c)$ of $\mp$ from $\S 10$ and ask which ones induce automorphisms of $\mathcal{G}(K)$. This amounts to determining whether $\Phi$ maps the relations (b) to relations. A computation shows that this happens exactly when

$$
a k-b r+c l \equiv 0 \bmod \Lambda
$$

where $\Lambda$ is the least common multiple of $(k, l),(k, r)$ and $(r, l)$. To determine which of these are strong automorphisms of $\mathcal{G}(K)$, we must check which sets $\left\{y^{b} z^{a}, x^{c}, 1\right\}$ are admissible i.e. satisfy (i), (ii) of $\S 5$ using the longitudes given by (a). Carrying out this computation gives the criterion

$$
a k-b r+c l=0 .
$$

An interesting observation that one makes during these calculations is that every special automorphism of $\mathcal{G}(K)$ perserves the preperipheral structure (see $\S 2$ ). Thus whenever two of the three linking numbers $k, l, r$ are nonzero there are such special automorphisms which are not strong. This suggests in light of Theorem 5 that there are 4-component links whose reduced structured groups are isomorphic, preserving the preperipheral structure, but not the peripheral structure.

I have not been able to construct such examples but we will be able to display below nonhomotopic links whose reduced structured groups are isomorphic via special isomorphisms.

12. We will need to record the $R$ and $A^{\prime}$ transformations for the commutator numbers of an element of $\mathcal{G}(K), K=L(k, l, r, d)$. This is done Table 3 .

\section{TABLE 3}

\begin{tabular}{ccccc}
4 & 5 & 6 & 7 & 8 \\
\hline$k$ & $r$ & 0 & $d$ & $d$ \\
$-k$ & 0 & $l$ & $-d$ & 0 \\
$-e_{1}$ & 0 & $e_{3}$ & $e_{5}$ & 0 \\
$e_{2}$ & $e_{3}$ & 0 & $e_{6}$ & $-e_{6}$ \\
0 & $-e_{1}$ & $-e_{2}$ & 0 & $e_{4}$ \\
$c e_{2}-b e_{1}$ & $-a e_{1}$ & 0 & $a e_{4}$ & $-a b e_{1}-c e_{6}+b e_{5}$
\end{tabular}

As before, the $t$ th column contains the differences $e_{t}^{\prime}-e_{t}$ where $\left\{e_{t}\right\} \mapsto\left\{e_{t}^{\prime}\right\}$ is the transformation determining a given row. 
The first two rows record the $R$-transformations corresponding to the relations (b) of $\S 11$. The next three rows record the $A$-transformations induced by a generating set of inner automorphisms and the last row records the $A^{\prime}$-transformation induced by $\Phi(a, b, c)$, somewhat simplified by composing with previous $A$-transformations.

It will also be useful to have the $R$ and $A$-transformations defined by commutators of the first five transformations of Table 3. These do not change $e_{i}, i \leq 6$ and so we record the effect on $e_{7}$ and $e_{8}$ in Table 4 . It will also be important to notice that the commutators of the last transformation with the first five are all consequences of these.

\begin{tabular}{cc}
\multicolumn{2}{c}{ TABLE } \\
7 & 8 \\
\hline$r$ & 0 \\
$l$ & $-l$ \\
0 & $k$ \\
0 & $e_{3}$ \\
$e_{1}$ & $-e_{1}$ \\
$e_{2}$ & 0
\end{tabular}

13. Before embarking on the proof of Theorem 9 we discuss some examples of this general classification in particular cases. A complete set of invariants of a 4-component link $L$ is the following:

(i) Integers: $k, l, r, e_{1}, e_{2}, e_{3}$ (the linking numbers of $L$ ).

(ii) Residue class of $d \bmod (k, l, r)$.

(iii) Commutator numbers $e_{4}, e_{5}, e_{6}, e_{7}, e_{8}$ up to $\rho$-equivalence explicitly given in Table 3, where $a, b, c$ satisfy $(d)$ of $\S 11$.

We point out the relation of these invariants with the $\bar{\mu}$-invariants so that we can compare the indeterminacies.

$$
\begin{aligned}
& \bar{\mu}_{12}=k, \quad \bar{\mu}_{13}=r, \quad \bar{\mu}_{23}=l, \quad \bar{\mu}_{14}=e_{1}, \quad \bar{\mu}_{24}=e_{2}, \quad \bar{\mu}_{34}=e_{3}, \\
& \bar{\mu}_{123}=d, \quad \bar{\mu}_{124}=e_{4}, \quad \bar{\mu}_{134}=e_{5}, \quad \bar{\mu}_{234}=e_{6}, \quad \bar{\mu}_{2134}=e_{7}, \quad \bar{\mu}_{3124}=e_{8} .
\end{aligned}
$$

The $\bar{\mu}$-invariants not contained in the above list can be expressed in terms of those in the list using the relations in [M1].

Recall from [M1] that the indeterminacy of $\bar{\mu}_{i_{1}, \ldots, i_{m}}$ is the greatest common divisor of all $\bar{\mu}_{j_{1}, \ldots, j_{n}}$ where $j_{1}, \ldots, j_{n}$ is obtained from $i_{1}, \ldots, i_{m}$ by deleting at least one index and permuting the remaining ones cyclically.

We will see that this indeterminacy is considerably reduced by our results.

Case 1. $k=l=r=e_{1}=e_{2}=e_{3}=0$.

In this case al values of $a, b, c$ define geometric automorphisms. From Table 3 one checks easily that these links are classified by the $\bar{\mu}$-invariants.

Case 2. $k=l=r=e_{1}=e_{2}=0, e_{3} \neq 0$.

Again all values of $a, b, c$ are allowed. From Table 3 one may check that a complete set of $\rho$-equivalence invariants are the following: $e_{3}, e_{4}, e_{5} \bmod e_{3}, e_{6} \bmod e_{3}$, $e_{7}$ and $e_{8} \bmod \left(d, e_{3}, e_{4}, e_{5}, e_{6}\right)$ and the quantity $\Delta$ defined by

$$
\Delta=e_{5} e_{6}-e_{3} e_{7} \bmod e_{3} \cdot\left(e_{4}, d\right) .
$$


(a)
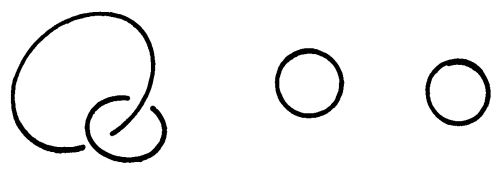

(b)

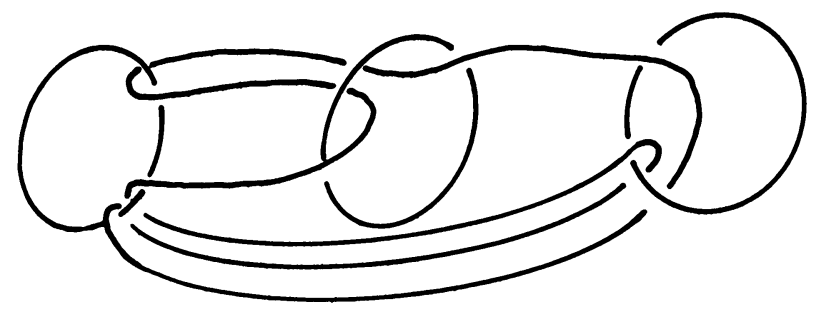

FIGURE 11

These coincide with the $\bar{\mu}$-invariants except for $\Delta$ which give an additional invariant. For example the links in Figure 11 have $d=e_{4}=e_{7}=e_{8}=0, e_{3}=1-$ therefore the same $\bar{\mu}$-invariants. However $e_{5}=e_{6}=0$ in (a) while $e_{5}=e_{6}=1$ in (b) - thus $\Delta=0$ in (a) and $\Delta=1$ in (b).

Case 3. $l=r=e_{1}=e_{2}=0$, but $k, e_{3} \neq 0$.

The geometric automorphisms require $a=0$, but $b$ and $c$ are arbitrary. From Table 3 we obtain the following set of $\rho$-equivalence invariants: $k, e_{3}, e_{4} \bmod k, e_{5}$ $\bmod e_{3}, e_{6} \bmod e_{3}, e_{7}$ and $e_{8} \bmod \left(d, k, e_{3}, e_{4}, e_{6}\right)$ and the integral quantity $\Delta^{\prime}$ defined by $\Delta^{\prime}=k e_{3} e_{7}-d e_{3} e_{4}-k e_{5} e_{6}$. These coincide with the $\bar{\mu}$-invariants, except for the new invariant $\Delta^{\prime}$.

These three cases give a complete set of homotopy invariants for any 4-component link with the property that each component has nonzero linking number with no more than one other component.

Case 4. $e_{1}, e_{2}$, and $e_{3}$ are pairwise relatively prime.

From Table 4 one can check that $e_{7}$ and $e_{8}$ can be transformed arbitrarily without affecting $e_{4}, e_{5}, e_{6}$. On the other hand, it can be checked from Table 3 that the quantity $\theta=e_{3} e_{4}-e_{2} e_{5}+e_{1} e_{6}$ is well defined modulo the greatest common divisor of $k e_{3}-r e_{2}, k e_{3}-l e_{1}$ and $a e_{1} e_{2}-b e_{1} e_{3}+c e_{2} e_{3}$ for all, $a, b, c$ satisfying $11(\mathrm{~d})$. The $\bar{\mu}$-invariants, except for the ordinary linking numbers, are completely indeterminate but $\theta$ is a complete invariant of $\rho$-equivalence.

Case 5. $k, l$ and $r$ are pairwise relatively prime.

Again $e_{7}$ and $e_{8}$ can be transformed arbitrarily. The quantity $\theta^{\prime}=r l e_{4}-k l e_{5}+$ $r k e_{6}$ is well defined modulo $l e_{1}-r e_{2}, l e_{1}-k e_{3}$ and this gives a complete invariant of $\rho$-equivalence. In this case the information carried by the $\bar{\mu}$-invariants is already contained in $\theta^{\prime}$. The strong automorphisms are all inner, as will be proved below in $§ 15$.

As an example of Case 4 or Case 5 consider the two links in Figure 12. We have $k=l=r=e_{1}=e_{2}=e_{3}=1, e_{i}=0$ for $i \geq 5$ for both links but $e_{4}=0$ in one case, 1 in the other. Thus the $\bar{\mu}$-invariants are the same but $\theta=0$ or 1 and $\theta^{\prime}=0$ or 1 , where both $\theta$ and $\theta^{\prime}$ are integers. It is of some interest to note, from Table 3 , that the special automorphism $\Phi(0,0,1)$, which is not strong, will identify the commutator numbers of these links. In fact the reduced groups of these links are 
isomorphic, via a special isomorphism extending $\Phi(0,0,1)$, although there does not seem to be such an isomorphism which preserves the preperipheral structure.

14. We now begin the proof that every $\Phi(a, b, c)$ which is a strong automorphism, i.e. $a, b, c$ satisfy $11(\mathrm{~d})$, is geometric. In case $k=r=l=0, K$ is almost trivial and the result follows from Theorem 8. If any two of $k, l, r$ are zero-say $k, l, 0$ - then we have to show $\Phi(1,0,0)$ and $\Phi(0,0,1)$ are geometric. But we can apply Theorem 7 , using the fact that the sublinks of $K$ formed by the first and second or second and third components are trivial.

Now we assume that at two of $k, r, l$ are nonzero. There will now be strong outer automorphisms to which we cannot apply Theorem 7 and so we will need another technique for constructing geometric automorphisms.

Our method will be the following. Suppose $\xi, \eta, \in \mathcal{G}(K)$ are represented by simple closed curves forming links $K_{\xi}, K_{\eta}$, when adjoined to $K$. If we know that $K_{\xi}$ and $K_{\eta}$ are homotopic, then, by Theorem 5, there is a geometric automorphism $\Phi$ of $\mathcal{G}(K)$ such that $\Phi(\xi)=\eta$. By examining Table 3 we may be able to relate $\Phi$ to a specific $\Phi(a, b, c)$. To establish that $K_{\xi}$ and $K_{\eta}$ are homotopic we will reorder the components in order to express them as $K_{\xi^{\prime}}^{\prime}, K_{\eta^{\prime}}^{\prime}$ corresponding to elements $\xi^{\prime}, \eta^{\prime} \in \mathcal{G}\left(K^{\prime}\right)$ where $K^{\prime}$ is some other common sublink of $K_{\xi}, K_{\eta}$. By previous results we may be able to find a geometric automorphism $\Phi^{\prime}$ of $\mathcal{G}\left(K^{\prime}\right)$ so that $\Phi^{\prime}\left(\xi^{\prime}\right)=\eta^{\prime}$.

To carry out this program we will need

Proposition 14. Let $K=L(k, l, r ; d)$ and $w \in \mathcal{G}(K)$ where

$$
w=x^{a} y^{b} z^{c}[x, y]^{e}[y, z]^{f} .
$$

Then $w$ can be represented (modulo commutators of weight 3 ) by a simple closed curve $\gamma$ so that, if $\gamma_{1}, \gamma_{2}, \gamma_{3}$ are the components of $K$ (in order) and $K^{\prime}$ is the link defined by $\gamma_{3}, \gamma, \gamma_{1}$ (in that order), then $K^{\prime}$ is isotopic to $L(c, a, r ; 0)$ and, under this isotopy, $\gamma_{2}$ represents $w^{\prime} \in \mathcal{G}\left(K^{\prime}\right)$, where $w^{\prime}=z t^{b} x^{k}[t, x]^{e}[x, z]^{d}[z, t]^{t}$ modulo commutators of weight 3. Here $x, y, z \in \mathcal{G}(K)$ and $z, t, x, \in \mathcal{G}\left(K^{\prime}\right)$ are the meridians of the peripheral structures defined in $\S 11$.

The proof consists of examining Figure 13, checking that $\gamma$ represents $w$, observing that Figure 14 is isotopic to Figure 13 and checking that $\gamma$ represents $w^{\prime}$.

We can rephrase Proposition 14 in terms of the commutator numbers as follows. If $L$ is a 4-component link with principal sublink $K=L(k, l, r ; d)$ and commutator numbers $\left\{e_{i}\right\}$, then the reordering of the components of $L$ defined by $(1,2,3,4) \mapsto$ $(3,4,1,2)$ yields a link $L^{\prime}$ with principal sublink $K^{\prime}\left(L\left(e_{3}, e_{1}, r ; 0\right)\right.$ and commutator numbers $\left\{e_{i}^{\prime}\right\}$ where $e_{1}^{\prime}=l, e_{2}^{\prime}=e_{2}, e_{3}^{\prime}=k, e_{4}^{\prime}=-e_{6}, e_{5}^{\prime}=-d$ and $e_{6}^{\prime}=e_{4} ; e_{7}^{\prime}$ and $e_{8}^{\prime}$ are not computed.

We will use a specific instance of this proposition.

COROllaRY 14. Suppose $K=L(k, r, l ; d)$ and $\xi, \eta \in \mathcal{G}(K)$ have commutator numbers $\left\{e_{i}\right\},\left\{\bar{e}_{i}\right\}$. Suppose $e_{i}=\bar{e}_{i}$ if $i=1,2,3,5$ and, for some integers $A, B$,

$$
\begin{aligned}
& \bar{e}_{4}-e_{4}=A\left(r e_{2}-l e_{1}\right) /(r, l), \\
& \bar{e}_{6}-e_{6}=B\left(k e_{3}-r e_{2}\right) /(k, r) .
\end{aligned}
$$

Also assume $e_{1}, e_{2}, e_{3}$ are pairwise relatively prime. Then the links $L_{\xi}$ and $L_{\eta}$, formed by adjoining curves representing $\xi, \eta$ to $K$, are homotopic. 

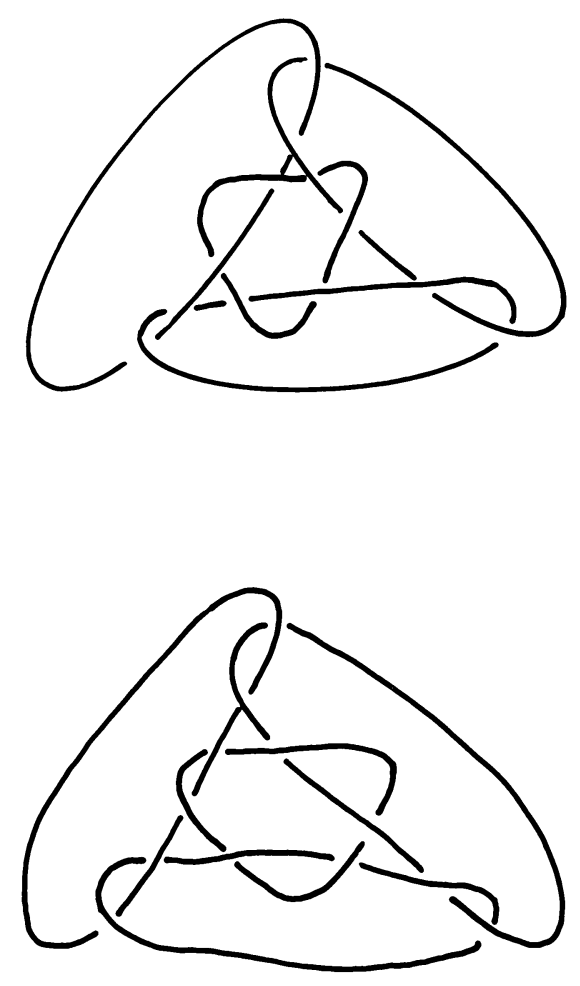

FIGURE 12

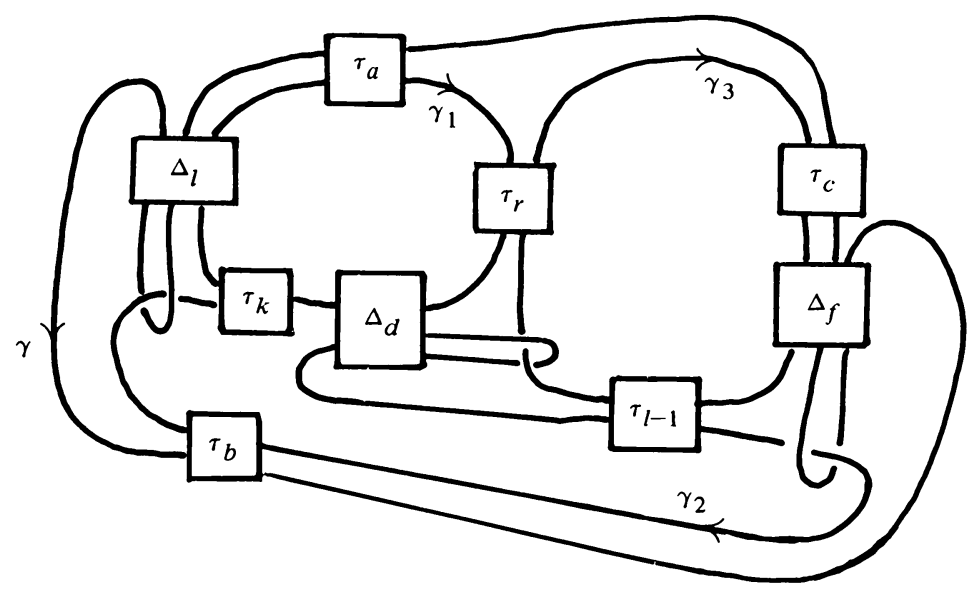

FIGURE 13

The point is that we could not derive this result from Table 3 without using some $\Phi(a, b, c)$. If we apply Proposition 14 to $L_{\xi}$ and $L_{\eta}$ we will see that their their new commutator numbers are equivalent using only the $R$-transformations and $A^{\prime}$ transformations defined by inner automorphisms i.e. the first 5 rows of Table 3 . We 


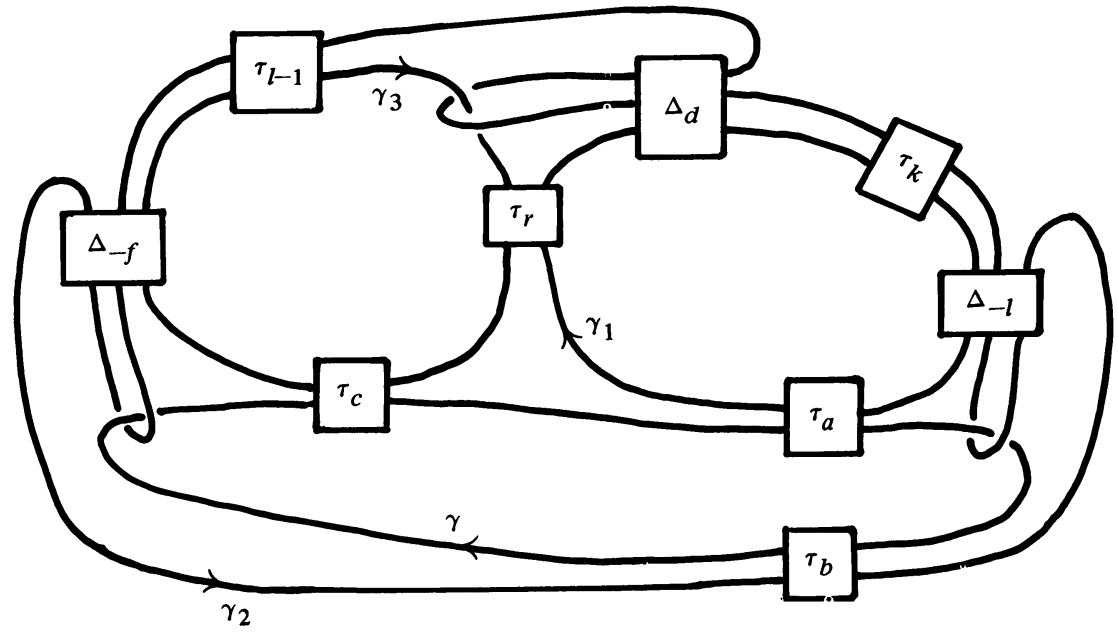

FIGURE 14

also use the fact that $e_{7}$ and $e_{8}$ are irrelevant, from Table 4 and the assumption that $e_{1}, e_{2}, e_{3}$ are pairwise relatively prime.

More specifically, renumbering the components of $L_{\xi}$ and $L_{\eta}$ give new links $L_{\xi}^{\prime}$, $L_{\eta}^{\prime}$ with the same principal sublink $K^{\prime}=L\left(e_{3}, e_{1}, r ; 0\right)$. The first 3 columns and 5 rows of Table 3 for $K^{\prime}$ are given in Table 5 .

\begin{tabular}{ccc}
\multicolumn{3}{c}{ TABLE } \\
4 & 5 & 6 \\
\hline$e_{3}$ & $r$ & 0 \\
$-e_{3}$ & 0 & $e_{1}$ \\
$-l$ & 0 & $k$ \\
$e_{2}$ & $k$ & 0 \\
0 & $-l$ & $-e_{2}$
\end{tabular}

If $\left\{e_{i}^{\prime}\right\}$ and $\left\{\bar{e}_{i}^{\prime}\right\}$ are commutator numbers of $L_{\xi}^{\prime}, L_{\eta}^{\prime}$ as specified by Proposition 14, then $\bar{e}_{5}^{\prime}=\bar{e}_{5}$ and

$$
\begin{aligned}
& \bar{e}_{4}^{\prime}-e_{4}^{\prime}=-B\left(k e_{3}-r e_{2}\right) /(k, r), \\
& \bar{e}_{6}^{\prime}-e_{6}^{\prime}=A\left(r e_{2}-l e_{1}\right) /(r, l) .
\end{aligned}
$$

Thus we may apply row $1-B k /(k, r)-A l /(r, l)$ times; row $2-A l /(r, l)$ times; row 4 $B r /(k, r)$ times; and row $5-A r /(r, l)$ times to transform $\left\{e_{i}^{\prime}\right\}$ to $\left\{\bar{e}_{i}^{\prime}\right\}$ for $i=4,5,6$. For $i=7,8$ we rewrite rows 2,3 and 6 of Table 4 for $K^{\prime}$ as in Table 6.

\begin{tabular}{cc}
\multicolumn{2}{c}{ TABLE } \\
7 & 8 \\
\hline$e_{1}$ & $-e_{1}$ \\
0 & $e_{3}$ \\
$e_{2}$ & 0
\end{tabular}

Using the fact that $e_{1}, e_{2}, e_{3}$ are pairwise relatively prime, it is not hard to see that these transformations can effect any desired change $\left(e_{7}^{\prime}, e_{8}^{\prime}\right) \mapsto\left(\bar{e}_{7}^{\prime}, \bar{e}_{8}^{\prime}\right)$.

Thus we conclude that $\left\{e_{i}^{\prime}\right\}$ and $\left\{\bar{e}_{i}^{\prime}\right\}$ are $\rho$-equivalent and so, by Theorem $6, L_{\xi}^{\prime}$ and $L_{\eta}^{\prime}$ are homotopic. 
15. Let us now consider the quotient group $S$ of strong automorphisms of $\mathcal{G}(K)$ modulo geometric automorphisms. We shall show that $S$ is trivial. The group of outer special automorphisms is abelian and, therefore, so is $S$. We can also see that $S$ is finite. Let $s$ be the greatest common divisor of $k, r, l$-we will prove that $s S=0$. In fact we can see that, for any strong $\Phi=\Phi(a, b, c) \Phi^{s}$ is inner. Modulo inner automorphisms $\Phi^{s}=\Phi(s a, s b, s c)$ and we can write

$$
s a=\gamma r-\sigma l, \quad s b=\tau l+\gamma k, \quad s c=\tau r+\sigma k,
$$

for suitable $\gamma, \sigma, \tau$ using $11(\mathrm{~d})$. One may then check that $\Phi(s a, s b, s c)$ is conjugation by

$$
w=x^{\tau r} y^{\tau l} z^{-\sigma l}[x, y]^{\tau d}[x, z]^{\sigma d}[y, z]^{-\gamma d-a \tau l} .
$$

It now suffices to show that $S=s S$, or, equivalently, $S=p S$ for every prime factor $p$ of $s$. If $\alpha, \beta, \gamma$ are the generators of $S$ represented by $\Phi(0, l /(r, l), r /(r, l))$, $\Phi(r /(k, r), k /(k, r), 0), \Phi(l /(k, l), 0,-k /(k, l))$, respectively, we shall show $\alpha, \beta, \gamma, \in$ $p S$.

We note the relation

$$
\frac{k s}{(k, r)(k, l)} \alpha-\frac{l s}{(k, l)(r, l)} \beta+\frac{r s}{(k, r)(l, r)} \gamma=0
$$

which holds because $(a, b, c) \rightarrow \Phi(a, b, c)$ defines a homomorphism of $Z \oplus \mathbf{Z} \oplus Z$ onto $S$. If $e(n)$ denotes the exponent of $p$ in the prime factorization of any integer $n(e(n)=\infty$ if $(p, n)=1)$, we may assume without loss of generality, that $e(k) \leq$ $e(r) \leq e(l)$. Then (a) implies tht $S / p S$ is generated by $\alpha, \beta$ (or $\beta, \gamma)$-so we only need show $\alpha, \beta \in p S$.

Consider the quantity $\theta=e_{3} e_{4}-e_{2} e_{5}+e_{1} e_{6}$ modulo $p$ (see $\S 13$, Case 4). Since $p \mid s$, it follows from Table 3 that $\theta$ is invariant under any $R$-transformation or $A^{\prime}$ transformation corresponding to an inner automorphism, and that $\alpha, \beta$ change $\theta$ by $e_{3} \sigma, e_{1} \tau$, respectively, where $\sigma=\left(r e_{2}-l e_{1}\right) /(r, l), \tau=\left(k e_{3}-r e_{2}\right) /(k, r)$.

Let $A, B$ be arbitrary integers and consider $\xi, \eta \in \mathcal{G}(K)$ with commutator numbers $\left\{e_{i}\right\},\left\{\bar{e}_{i}\right\}$, and $\theta, \bar{\theta}$ the invariants of the preceding paragraph, where $e_{i}=\bar{e}_{i}$ for $i \leq 3, e_{i}^{\prime}=0$ for $4 \leq i \leq 6, e_{4}=A_{1} \sigma, e_{5}=0$ and $e_{6}=B_{2} \tau$. If $e_{1}, e_{2}, e_{3}$ are pairwise relatively prime it follows from Corollary 14 that $L_{\xi}$ and $L_{\eta}$ are homotopic. Now $\bar{\theta} \equiv 0$ and $\theta \equiv A e_{3} \sigma+B e_{1} \tau \bmod p$ and there must be a geometric automorphism that sends $\theta$ and $\bar{\theta}$. This implies that $C \alpha+D \beta \in p S$ for some $C, D$ satisfy

$$
C e_{3} \sigma+D e_{1} \tau \equiv A e_{3} \sigma+B e_{1} \tau \bmod p .
$$

We will show that a suitable collection of $\left(e_{1}, e_{2}, e_{3}, A_{1}, B\right)$ will produce enough values of $(C, D)$ to show that $\alpha, \beta \in p$.

(i) $e_{1}=p, e_{2}=e_{3}-1, A=1$ : From (b) we conclude $C \equiv 1 \bmod p$.

Therefore $\alpha \equiv q^{\prime} \beta \bmod p S$ for some $q^{\prime}$.

(ii) $e_{1}=e_{2}=1, e_{3}=p, B=1$, if $e(k)=e(r)$ : From (b) we conclude $D \equiv 1$ $\bmod p$ and so $\beta \equiv q \alpha \bmod p S$, for some $q$.

(iii) $e_{1} \equiv r /(r, l) \bmod p, e_{2} \equiv l /(r, l) \bmod p, e_{3}=1, B=1$, if $e(k)<e(r)$ : We obtain $D \equiv 1 \bmod p$ and so $\beta \equiv q \alpha \bmod p S$, for some $q$. Note that we can choose $e_{1}, e_{2}, e_{3}$ pairwise relatively prime by the Dirichlet Theorem.

If $q \equiv 0 \bmod p$ in (ii) or (iii), then, together with (i), this finishes the proof. We now assume $q \not \equiv 0 \bmod p$. 
(iv) Choose $e_{1}, e_{2}, e_{3}$ to satisfy $e_{1} \tau \equiv 1, e_{3} \sigma q \equiv 1 \bmod p, A=0, B=1$ : From (b) we have $C+D q \equiv q \bmod p$. Therefore $C \alpha+D \beta \equiv q(1-D) \alpha+D q \alpha \equiv q \alpha \in p S$.

This complete the argument if we can show that $e_{1}, e_{2}, e_{3}$ can be chosen to satisfy the requirements of (iv) and be pairwise relatively prime. The equations to be satisfied are

$$
\begin{gathered}
\frac{k}{(k, r)} e_{1} e_{3}-\frac{r}{(k, r)} e_{2} e_{2} \equiv 1 \quad \bmod p, \\
q \frac{r}{(r, l)} e_{2} e_{3}-q \frac{l}{(r, l)} e_{1} e_{3} \equiv 1 \quad \bmod p .
\end{gathered}
$$

If $e(k)<e(r)$ we may set $e_{3}=1$ and solve for $e_{1}$ and $e_{2}$. Since $e_{1} \not \equiv 0 \bmod p$, we may use the Dirichlet Theorem to choose $\left\{e_{i}\right\}$ pairwise relatively prime. If $e(r)=e(l)$, we may set $e_{2}=0, e_{3}=1$ and solve for $e_{1} ; e_{1} \not \equiv 0 \bmod p$ and so we can achieve pairwise relatively prime. If $e(k)=e(r)<e(l)$ and $p \neq 2$, then we may choose $e_{2}, e_{3}$ to satisfy the second equation and $(k /(k, r)) e_{3}-(r(k, r)) e_{2} \equiv 0 \bmod p$ and, therefore, solve the first equation for $e_{1}$. This is impossible when $p=2$.

Thus we are finished unless $p=2, e(k)=e(r)<e(l)$. If $l=0$, we can finish the proof at this point by invoking Theorem 7 to conclude that $\Phi(0,0,1)$ is geometric and so $\alpha=0$. Write

16. To complete the proof when $p=2$, we consider $\xi, \eta \in \mathcal{G}(K)$ with commutator numbers $\left\{e_{i}\right\}$ and $\left\{\bar{e}_{i}\right\}$ defined by

$$
\begin{aligned}
& e_{1}=1, \quad e_{2}=0, \quad e_{3}-2^{n}, \quad e_{4}=1, \quad e_{5}=0, \quad e_{6}=1, \quad e_{7}=e_{8}=0 \\
& \bar{e}_{i}=e_{i} \quad \text { for } i \leq 7, \quad \bar{e}_{8}=1 .
\end{aligned}
$$

We rewrite Table 3 as in Table 7, using these values of $e_{1}, e_{2}, e_{3}$.

\begin{tabular}{ccccc}
\multicolumn{5}{c}{ TABLE 7} \\
4 & 5 & 6 & 7 & 8 \\
\hline-1 & 0 & $2^{n}$ & $e_{5}$ & 0 \\
0 & -1 & 0 & 0 & $e_{4}$ \\
0 & 0 & $l$ & 0 & $r e_{4}-d$ \\
0 & 0 & $2^{n} k$ & $k e_{5}$ & $r e_{4}$ \\
0 & 0 & 0 & $e_{6}$ & $2^{n} e_{4}-e_{6}$ \\
0 & 0 & $-2^{n} b$ & 0 & $-a b-c e_{6}$
\end{tabular}

We can rewrite Table 4 as in Table 8.

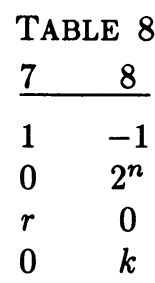

We will show that $L_{\xi}$ and $L_{\eta}$ are homotopic. Thus there is a geometric automorphism of $\mathcal{G}(K)$ which does not change $e_{i}$, for $i \leq 7$, but changes $e_{8}$ by one. Since Table 8 contains all commutators of those in Table 7, we can assume that any sequence of transformations be applied in the form

$$
T_{1}^{a_{1}} T_{2}^{a_{2}} T_{3}^{a_{3}} T_{4}^{a_{4}} T_{5}^{a_{5}} T_{6}^{a_{6}} T
$$


where $T_{i}$ is the transformation corresponding to the $i$ th row of Table 7 and $T$ is a sequence of transformations from Table 8.

Since $e_{4}, e_{5}, 4_{6}$ are not changes we see that $a_{1}=a_{2}=0$. Now using the transformations of Table 8 we can replace some of those in Table 7 by simpler ones and consider a new table of transformations of $e_{6}, e_{7}, e_{8}$ (Table 9).

TABLE 9

\begin{tabular}{ccc}
6 & 7 & 8 \\
\hline$l$ & 0 & $-d$ \\
$2^{n} k$ & 0 & 0 \\
$-2^{n} b$ & 0 & $-a b-c e_{6}$ \\
0 & 1 & -1 \\
0 & 0 & $2^{n}$ \\
0 & 0 & $r$ \\
0 & 0 & $k$
\end{tabular}

The last four rows generate the commutators and $T_{1}, T_{2}, T_{5}$ have been eliminated.

If we assume $n$ is large, then $a_{3}$ must be even. Since $e_{7}$ is not changed, the fourth row of Table 9 cannot be used. Now all the entries in the $e_{8}$ column are even except possibly for the first row, which must be used an even number of times, and the third row. Since $e_{8}$ is changed by 1 , some choice of $\Phi(a, b, c)$ must be used in which $-a b-c e_{6}$ is odd. The initial value of $e_{6}$ is 1 and every transformation of Table 7 changes it by an even amount; therefore $e_{6}$ will alway be odd. We conclude that some $\Phi(a, b, c)$ is geometric for which $-a b-c$ is odd. This quantity is odd for both $\alpha$ and $\beta(e(k)=e(r))$ and so $A \alpha+B \beta \in 2 S$, for some $A, B$ satisfying $A+B$ odd. Since we have already proved in $\S 15$, that $\alpha \equiv \beta \bmod 2$, we conclude that $\alpha \in 2 S$.

17. We finally have to prove that $L_{\xi}$ and $L_{\eta}$ of $\S 16$ are homotopic. Using Proposition 14 we can replace $L_{\xi}, L_{\eta}$ by $L_{\xi}^{\prime}, L_{\eta}^{\prime}$ with principal sublink $K^{\prime}=L\left(2^{n}, 1, r ; 0\right)$ and the same commutator numbers $\left\{e_{i}^{\prime}\right\}$, for $i \leq 6$, given by $e_{1}^{\prime}=l, e_{2}^{\prime}=0, e_{3}^{\prime}=k$, $e_{4}^{\prime}=-1, e_{5}^{\prime}=-d, e_{6}^{\prime}=1$.

From the tables of transformations for $K^{\prime}$ derived from Tables 3 and 4 we extract Table 10.

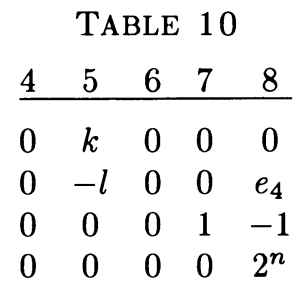

The first two rows come from rows 4 and 5 of Table 3 and the last two rows come from rows 2 and 3 of Table 4 . If we apply the first row of Table $10 l /(k, l)$ times and the second row $k /(k, l)$ times we obtain a new transformation:

$$
\left(\begin{array}{lllll}
0 & 0 & 0 & 0 & \left.k e_{4} /(k, l)\right)
\end{array}\right)
$$

We now restrict ourselves to this transformation and the last two rows of Table 10, which only change $e_{7}$ and $e_{8}$. Since $e_{4}=-1$ we obtain Table 11 . 


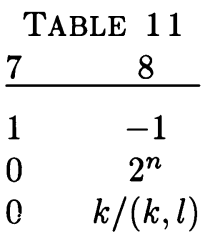

Now $k /(k, l)$ is odd and it is clear that these three transformations can effect any desired change in $e_{7}$ and $e_{8}$. This shows that $L_{\xi}^{\prime}$ and $L_{\eta}^{\prime}$ are homotopic and the proof is now complete.

\section{REFERENCES}

[FR] R. Fenn and D. Rolfsen, Spheres may link homotopically in 4-space, J. London Math. Soc. (2) 34 (1986), 177-184.

[G] C. Griffen, New results on link equivalence relations, preprint, 1976.

[G1] _ Link concordance implies link homotopy, Math. Scand. 45 (1979), 243-254.

[GO] D. Goldsmith, Concordance implies homotopy for classical links in $\mathrm{M}^{3}$, Comment. Math. Helv. 54 (1979), 347-355.

[H] S. Humphries, On weakly distinguished bases and free generating sets of free groups, Quart. J. Math. 36 (1985), 215-220.

[HS] F. Hosokawa and S. Suzuki, Linking 2-spheres in the 4-sphere, preprint.

[KO] K. H. Ko, Seifert matrices and boundary link cobordism, Ph.D. Thesis, Brandeis University, 1984.

[L] J. Levine, Surgery on links and the $\bar{\mu}$-invariants, Topology 26 (1987), 45-61.

[LS] R. Lyndon and P. Schupp, Combinatorial group theory, Springer-Verlag, New York, 1977.

[M] J. Milnor, Link groups, Ann. of Math. (2) 59 (1954), 177-195.

[M1] _ , Isotopy of links, Algebra, geometry and topology (A symposium in honor of S. Lefschetz), Princeton Univ. Press, Princeton, N.J., 1957, pp. 280-306.

[MR] W. Massey and D. Rolfsen, Homotopy classification of higher dimensional links, Indiana Univ. Math. J. 34 (1985), 375-391.

[MKS] W. Magnus, A. Karrass, and D. Solitar, Combinatorial group theory, Dover, New York, 1976.

[P] A. Plans, Contribution to the homotopy of systems of knots, Rev. Mat. Hisp. Amer. 17 (1957), 224-237 (Spanish).

[R] D. Rolfsen, Isotopy of links in codimension two, J. Indian Math. Soc. 36 (1972), 978-984.

[S] J. P. Serre, Lie algebras and Lie groups, Benjamin, New York, 1965.

[St] J. Stallings, Homology and central series groups, J. Algebra 2 (1965), 170-181.

[T] A. Tristram, Some cobordism invariants for links, Proc. Cambridge Philos. Soc. 66 (1969), 251-264.

[FL] M. Freedman and X. S. Lin, On the $(A, B)$-slice problem, preprint.

[Li] X. S. Lin, On equivalence relations of links in 3-manifolds, preprint.

Department of Mathematics, Brandeis University, Waltham, MassachuSETTS 02254 